\title{
Thermischer Einfluss urbaner Untergrundstrukturen auf die Grundwassertemperaturen im Kanton Basel-Stadt
}

\author{
Dominic Becker ${ }^{1} \cdot$ Jannis Epting ${ }^{1}$ iD \\ Eingegangen: 13. Oktober 2020 / Überarbeitet: 15. Februar 2021 / Angenommen: 21. April 2021 / Online publiziert: 27. Mai 2021 \\ (c) Der/die Autor(en) 2021
}

\section{Zusammenfassung}

In Basel $(\mathrm{CH})$ wurde mit Monitoringsystemen der thermische Einfluss unterschiedlicher Untergrundstrukturen, einschließlich fünf Tiefgaragen sowie einem Autobahntunnel, auf die urbanen Grundwasserressourcen untersucht. Die Daten wurden anschließend mit gemessenen Meteo- und Grundwassertemperaturdaten sowie Resultaten einer Wämetransportmodellierung zusammenhängend ausgewertet.

In den Tiefgaragen wurden auch über die Wintermonate deutlich erhöhte durchschnittliche Temperaturen zwischen 18,8 und $21,1^{\circ} \mathrm{C}$ erfasst. Über den weitaus größten Zeitraum emittieren die Tiefgaragen somit Wärme in den Untergrund. Die Messdaten im Autobahntunnel hingegen deuten darauf hin, dass in den Sommermonaten zwar auch Wärme in den Untergrund emittiert, im Winterhalbjahr aber Wärme aus dem Untergrund absorbiert wird.

Zudem zeigen die Temperaturverläufe in den Tiefgaragen eine klare Abhängigkeit von der Nutzungsart: bei höherem Aufkommen täglicher Ein- und Ausfahrten konnten größere tägliche Temperaturanstiege nachgewiesen werden, mit Unterschieden von bis zu $2{ }^{\circ} \mathrm{C}$ in den Tagesmittelwerten. Besonders deutlich wird dies im Zeitraum des „Lockdowns“ während der COVID-19-Pandemie zwischen März und Mai 2020.

Schlüsselwörter Grundwassererwärmung · Temperaturmessungen · Tiefgaragen und Tunnelbauwerke · Wärmelasten Untergrundstrukturen · COVID-19-Pandemie

\section{Thermal impact of subsurface urban structures on groundwater temperatures in the city of Basel}

\begin{abstract}
In Basel $(\mathrm{CH})$, the thermal impact of various subsurface structures on urban groundwater resources, including five underground parking lots and a freeway tunnel, were investigated by monitoring systems. Data were analyzed together with meteorological and groundwater temperature data and results from heat-transport modelling.

Significantly elevated temperatures between 18.8 and $21.1^{\circ} \mathrm{C}$ were recorded in the underground parking lots, even in winter. Thus, underground parking lots emit heat into the surroundings all year. In comparison, data recorded in the freeway tunnel indicate that in the winter months heat can also be absorbed from below ground.

In addition, the temperatures of underground parking lots show a clear dependence on the type of use: with a higher number of daily entrances and exits, greater daily temperature increases were detected, with differences of up to $2^{\circ} \mathrm{C}$. This became particularly clear in the "lockdown" period during the COVID-19 pandemic between March and May 2020.
\end{abstract}

Keywords Elevated groundwater temperatures - Temperature measurements - Underground parking and tunnels · Thermal loads of subsurface structures $\cdot$ COVID-19 pandemic

Jannis Epting

jannis.epting@unibas.ch

1 Angewandte und Umweltgeologie, Departement

Umweltwissenschaften, Universität Basel,

Bernoullistr. 32, 4056 Basel, Schweiz 


$\begin{array}{ll}\text { Abkürzungen } \\ A_{G W} & \text { Gebäudefläche im Kontakt mit dem Grundwasser } \\ \text { BAES } & \text { Basel Aeschenplatz } \\ \text { BKLI } & \text { Basel Klingelbergstrasse } \\ E & \text { Wärmeaustausch } \\ \mathrm{GW} & \text { Grundwasser } \\ \mathrm{GWM} & \text { Grundwassermessstelle } \\ k & \text { Wärmedurchgangskoeffizient } \\ \mathrm{SUHI} & \text { Subsurface Urban Heat Island } \\ \mathrm{T}_{\mathrm{GW}} & \text { Grundwassertemperatur } \\ \mathrm{T}_{\mathrm{GW}} & \text { simulierte Grundwassertemperatur } \\ \mathrm{T}_{\mathrm{TG}} & \text { Temperatur der Tiefgaragenmessungen } \\ \mathrm{T}_{\text {meteo }} & \text { meteorische Temperatur } \\ \Delta \mathrm{T} & \text { Temperaturdifferenz }\end{array}$

\section{Einleitung}

Untersuchungen zu erhöhten urbanen Grundwassertemperaturen und der Subsurface Urban Heat Island (SUHI)Effekt sind mittlerweile ein etablierter Forschungsbereich (z. B. Ferguson und Woodbury 2007; Zhu et al. 2010; Menberg et al. 2013a; Epting and Huggenberger 2013). Es existieren jedoch nur wenige Arbeiten, welche eine differenzierte Betrachtung des thermischen Einflusses von Untergrundstrukturen behandeln. Eine Kombination von verschiedenen Einflussfaktoren wie die zunehmende Bodenversiegelung und die thermische Nutzung des Untergrundes, aber vor allem auch wärmeemittierende Untergrundstrukturen wie Tunnelbauten und Tiefgaragen resultieren in einem nachweislichen Anstieg der basel-städtischen Grundwassertemperaturen von bis zu $9{ }^{\circ} \mathrm{C}$ (Epting et al. 2013, 2017a, b; Mueller et al. 2018). Vorausgehende Analysen, die in der Stadt Basel (Schweiz) durchgeführt wurden, haben bereits gezeigt, dass in urbanen Gebieten der anthropogene thermische Einfluss beheizter Untergrundstrukturen größer ist als die zu erwartenden Auswirkungen des Klimawandels (Epting und Huggenberger 2013).

Stadtentwicklung wird zunehmend auch im Untergrund stattfinden (z.B. Bobylev 2009), thermische Beeinträchtigungen urbaner Untergrundressourcen werden vermehrt zu Konflikten zwischen den verschiedenen Nutzern und thermische Kontaminationen werden zwangsläufig zu einer Verringerung der Grundwasserqualität führen (z. B. Possemiers et al. 2014). Doch bisher ist wenig über die biologischen, chemischen und physikalischen Aspekte des Grundwassers und über den Einfluss erhöhter Temperaturen auf die Grundwasserqualität bekannt (CEC 2000; Bates et al. 2008; Brielmann et al. 2009; Jesußek et al. 2013; Kipfer und Livingstone 2008). Die Auswirkungen von SUHI auf die Untergrundtemperaturen entwickeln sich somit zu einem globalen Grundwasserqualitätsproblem.
Der Wärmeverlust von Untergrundstrukturen über das Erdreich kann bis zu 50\% der jährlichen Wärmelast eines Gebäudes betragen (z. B. Deru 2003). Mit zunehmend effizienter werdender oberirdischer Gebäudeisolation gewinnt eine detaillierte Betrachtung unterirdischer Wärmeverluste vermehrt an Bedeutung. Bereits in vorausgegangenen SUHI-Studien wurden Tiefgaragen als Quellen für Anomalien der Grundwassertemperaturen diskutiert (Epting 2017; Iskander et al. 2001; Menberg et al. 2013b; Tissen et al. 2019; Zhu et al. 2010).

Im Allgemeinen berücksichtigen die meisten Untersuchungsansätze nur einzelne unterirdische Strukturen. So verwendete Ampofo et al. (2006) beispielsweise numerische Modelle, um die Wärmelast einer U-Bahn zu untersuchen, wobei bis zu 30\% der Wärmelast der U-Bahn in den Untergrund eingetragen werden kann. Dědeček et al. (2012) zeigten, dass für zwei Orte in Mitteleuropa (PragSpořilov/Tschechien und Šempeter/Slowenien) das thermische Regime im Untergrund sowohl durch die jüngsten regionalen Klimaveränderungen als auch durch thermische Effekte lokaler anthropogener Strukturen stark beeinflusst wird. Obwohl Dahlem (2000) bereits feststellte, dass der Wärmeverlust durch die advektive Grundwasserströmung im Vergleich zu rein konduktiven Wärmeverlusten einen Faktor 10 ausmachen kann, untersuchten nur wenige Studien den Einfluss der Grundwasserströmung auf den Wärmeverlust beheizter Gebäudestrukturen. Auf städtischer Ebene haben Menberg et al. (2013a) und Benz et al. (2015) analytische Wärmeflussmodelle und einen GIS-Ansatz (Geographisches Informationssystem) verwendet, um zu zeigen, dass Untergrundstrukturen einen signifikanten Anteil der gesamten anthropogenen Wärmelast von urbanen Grundwasserleitern ausmachen. Epting et al. (2017a) präsentierten eine systematische Bewertung der thermischen Auswirkungen von unterirdischen Gebäudestrukturen auf städtische Grundwasserressourcen. Ein wesentliches Fazit dieser Arbeit war, dass thermische Auswirkungen von Untergrundstrukturen gemeinhin unterschätzt werden, v. a. auch wegen eines Mangels an Informationen und zuverlässigen Daten.

Im Rahmen der Untersuchungen wurde der thermische Einfluss von urbanen Untergrundstrukturen im Stadtgebiet von Basel evaluiert. Der Fokus lag dabei auf Tiefgaragen und Tunnelbauwerken, welche in die grundwassergesättigte Zone hineinragen und somit einen direkten thermischen Einfluss auf das Grundwasser haben. Die über 1 Jahr kontinuierlich aufgezeichneten Daten der Temperaturen in den Tiefgaragen $\mathrm{T}_{\mathrm{TG}}$ und dem Autobahntunnel werden im Zusammenhang mit meteorischen Temperaturdaten $\left(\mathrm{T}_{\text {meteo }}\right)$ zweier Wetterstationen, Temperaturdaten $\left(\mathrm{T}_{\mathrm{GW}}\right)$ von neun Grundwassermessstellen (GWM) sowie Resultaten einer Wämetransportmodellierung $\left(\mathrm{T}_{\mathrm{GWsim}}\right)$ ausgewertet. 


\section{Untersuchungsgebiet}

Die untersuchten Untergrundstrukturen liegen im Stadtgebiet von Basel $\left(24 \mathrm{~km}^{2}\right)$. Der Kanton Basel-Stadt befindet sich im Nordwesten der Schweiz auf ca. $260 \mathrm{~m}$ ü. M., grenzt im Norden an Deutschland und Frankreich und im Süden an die Ausläufer des Falten- und Tafeljuras. Der Rheingraben als alluvialer Ablagerungsraum umfasst die pleistozänen Niederterrassenschotter des Rheins, der Wiese und der Birs, welche auf wasserstauendem Septarienton und Elsässer Molasse liegen (Bitterli-Brunner und Fischer 1988). Die Grundwassermächtigkeiten liegen zwischen 4 und $12 \mathrm{~m}$, der Flurabstand beträgt zwischen 5 und $12 \mathrm{~m}$, und Grundwasserfließgeschwindigkeiten liegen im Bereich von 0,04 und $4 \mathrm{md}^{-1}$ (Epting et al. 2017b). Die regionale Grundwasserfließrichtung folgt dem hydraulischen Gradienten in Richtung der Vorfluter Rhein, Birs, Birsig und Wiese. Es herrschen also größtenteils Grundwasser-exfiltrierende Verhältnisse; während Hochwasserereignissen kann aber auch Oberflächenwasser in den Grundwasserleiter infiltrieren (Abb. 1).

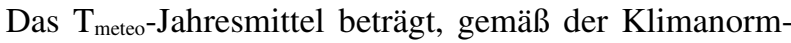
werte des Zeitraumes 1981 bis $201010,5^{\circ} \mathrm{C}$, allerdings mit steigender Tendenz, sodass mit $12,3^{\circ} \mathrm{C}$ im Jahr 2019 das Maximum seit Aufzeichnungsbeginn verzeichnet wurde; die mittlere Jahresniederschlagssumme beträgt rund $850 \mathrm{~mm}$ (Basel-Stadt 2020; Klimanormwerte 1981-2010: Lufttemperatur $2 \mathrm{~m}$, MeteoSchweiz; 1981-2010: Niederschlagssumme, MeteoSchweiz). Damit zählt Basel zu einer der wärmsten und niederschlagsärmeren Regionen der Schweiz.

\section{Methodik}

\section{Gebäudestrukturen im Untergrund von Basel}

Auf Grundlage von vorausgehenden Arbeiten und der systematischen Dokumentation von Gebäudestrukturen im Untergrund von Basel (Epting et al. 2017a) konnten insgesamt fünf Tiefgaragen, welche in die grundwassergesättigte Zone reichen, ausgewählt werden (Abb. 1 und Tab. 1). Die in einem GIS organisierten Daten von 3016 Gebäudestrukturen im Untergrund von Basel enthalten sowohl Informationen zu den Geometrien der Untergrundstrukturen, der Gebäudenutzung als auch zur mittleren und maximalen Grundwasserhöhe aus Messungen der letzten 20 Jahre. Ebenfalls wurden die Differenzen zwischen den Grundwasserhöhen und Unterkantentiefen an den jeweiligen Standorten der Untergrundgebäudestrukturen erfasst.

Neben den Messungen von $\mathrm{T}_{\mathrm{TG}}$ konnten auch Messdaten aus den Autobahntunneln der Nordtangente in die Untersuchungen mit einbezogen werden. Insgesamt wur- den hier an sechs Messstandorten Temperaturdaten der Tunnelinnenluft aufgezeichnet. Jeweils zwei Messsensoren an der Stammlinie (Fahrtrichtung Deutschland nach Frankreich und Frankreich nach Deutschland), eine am Tunnelportal des St. Johanns-Tunnels (SJT; Fahrtrichtung Frankreich nach Deutschland), zwei an Auf- und Abfahrt des Tunnelanschlusses Luzernerring (TLR) und eine an der Stammlinie des Horburgtunnels auf der Kleinbasler Rheinseite (Fahrtrichtung Deutschland nach Frankreich). Etwa $2 \mathrm{~km}$ der Tunnelstrecke liegen in der grundwassergesättigten Zone (Abb. 1).

\section{Messinstrumente}

Die Erfassung der Temperaturdaten erfolgte mit fünf Datenloggern des Typs EA WLAN-TH+ sowie einem EA WLAN-T+ der Marke Display Visions der Firma Electronic Assembly GmbH aus Gilching, Deutschland. Die Messsysteme erfassen die Temperatur in zehnsekündigen bis zwölfstündigen Messintervallen mit einer Genauigkeit von $\pm 0,2^{\circ} \mathrm{C}$ und einer Auflösung von $0,01^{\circ} \mathrm{C}$. Die Verarbeitung der Daten wurde durch das Programm EasyLog WiFi von Lascar electronics vorgenommen, welches die Visualisierung und den Export der Daten als Microsoft ExcelTabelle oder als Text-Datei (CSV) ermöglichte.

Für die Tiefgaragen wurden kontinuierlich über die Dauer eines Jahres zwischen Dezember 2019 und Dezember 2020 stündliche Mittelwerte der Temperaturen aufgezeichnet. Im Autobahntunnel Nordtangente in Basel wurden an den sechs Messstandorten mit den gleichen Messsystemen und der gleichen Auflösung von Dezember 2018 bis November 2019 Temperaturen aufgezeichnet.

\section{D-Simulation der Grundwasserströmung und -temperatur}

Eine weitere Grundlage für die Auswertungen sind 3DSimulationen der Grundwasserströmung und des Wärmetransports (FEFLOW; Diersch 2014), welche im Rahmen des BFE-Projektes (Schweizer Bundesamt für Energie) ,IstZustand und Temperatur-Entwicklung Schweizer Lockergesteinsgrundwasservorkommen" stattgefunden haben und unter anderem in Mueller et al. (2018) publiziert wurden. Im Rahmen dieses Projektes wurde für das Stadtgebiet von Basel, basierend auf hochaufgelösten tiefendifferenzierten Temperaturmessungen und 3D-Simulationen, der Istzustand des hydraulischen und thermischen Grundwasserregimes für den Zeitraum von 2010 bis 2015 abgeleitet (Abb. 1). Für die hier vorgestellten Auswertungen wurden die Simulationsresultate der $\mathrm{T}_{\mathrm{GW} s i m}$ an den jeweiligen Koordinaten inkl. Tiefe der Messstandorte extrahiert. 
Abb. 1 a Standorte der Tiefgaragen (1 - Storchen; 2 Clarastrasse; 3 - St. Claraspital; 4 - St. Jakob Park; 5 Anfos), des Tunnelbauwerkes Nordtangente, der ausgewählten Grundwassermessstellen und der Meteostationen BKLI und BAES im Untersuchungsgebiet. Dargestellt sind zudem die mittleren simulierten Grundwassertemperaturen $\left[{ }^{\circ} \mathrm{C}\right]$ und das hydrogeologische Regime für die Jahre 2010-2015 (nach Mueller et al. 2018). b Charakteristisches hydrogeologisches Profil durch den urbanen Grundwasserkörper

Fig. 1 a Locations of the underground parkings (1 - Storchen; 2 -Clarastrasse; 3 -St. Claraspital; 4 - St. Jakob Park; 5 - Anfos), the freeway tunnel Nordtangente, the selected groundwater observation wells and the meteorological stations BKLI and BAES in the study area. The mean simulated groundwater temperatures $\left[{ }^{\circ} \mathrm{C}\right]$ and the hydrogeological regime for the years 2010-2015 (according to Mueller et al. 2018) are also shown. b Characteristic hydrogeologic profile through the urban aquifer

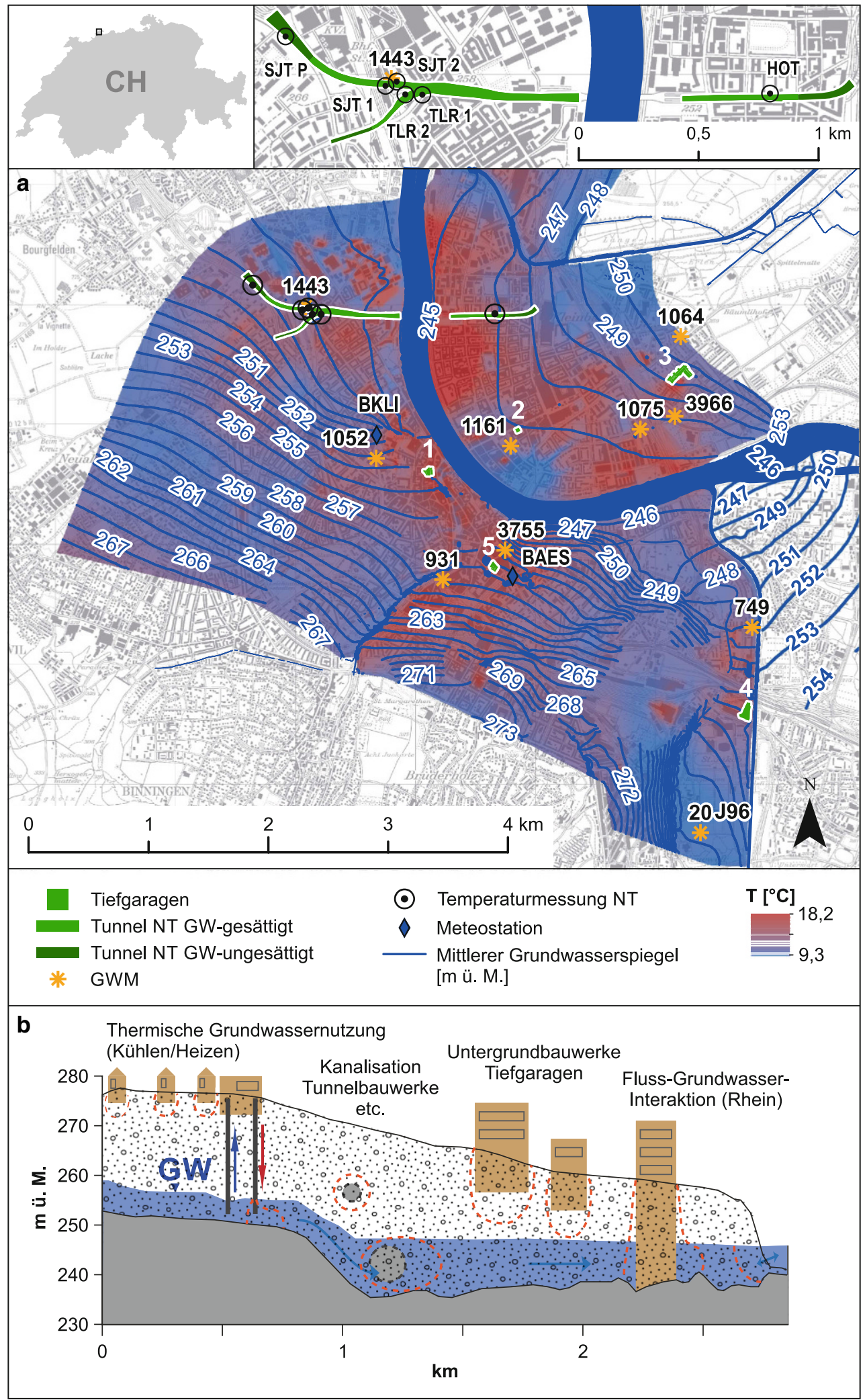


Tab. 1 Standortbeschreibung der Messsysteme in den Tiefgaragen (1-5) sowie Auswahl der Grundwassermessstellen Table 1 Monitoring systems in the underground parkings (1-5) and selection of groundwater observation wells

\begin{tabular}{|c|c|c|c|c|}
\hline \multirow[t]{4}{*}{ Standort } & $\begin{array}{l}\text { Tiefe Messystem } \\
\text { unter GOK }[\mathrm{m}]\end{array}$ & $\begin{array}{l}\text { Gebäude- } \\
\text { grundfläche } \\
{\left[\mathrm{m}^{2}\right]}\end{array}$ & GWM & \multirow[t]{4}{*}{ Settings und Nutzung } \\
\hline & GOK [m ü. M.] & $\begin{array}{l}\text { Kontaktfläche } \\
\text { Untergrund } \\
{\left[\mathrm{m}^{2}\right]}\end{array}$ & GW-Zustrom & \\
\hline & $\begin{array}{l}\text { Mittlerer GW- } \\
\text { Stand [m ü. M.] }\end{array}$ & $\begin{array}{l}\text { Kontaktfläche } \\
\text { GW }\left[\mathrm{m}^{2}\right]\end{array}$ & GW-Abstrom & \\
\hline & $\begin{array}{l}\text { Gebäudetiefe [m } \\
\text { ü. M.] }\end{array}$ & & & \\
\hline \multirow{4}{*}{$\begin{array}{l}\text { 1: Parkhaus } \\
\text { Storchen } \\
\text { 2'611'109/1'267'605 }\end{array}$} & $-5,1$ & $1 ' 574$ & Bernoullianum (1052) & \multirow{4}{*}{$\begin{array}{l}\text { Grossbasler Innenstadt nahe Rheinufer } \\
\text { 00:00-24:00 Uhr: Wochen-, Sonn- und Feiertage } 142 \\
\text { Kurzzeitstellplätze; während Ladenöffnungszeiten } \\
\text { (Mo-Sa) komplett belegt; } 800 \text { täglichen Ein- und } \\
\text { Ausfahrten }\end{array}$} \\
\hline & 255,4 & $44^{\prime} 768$ & 2'610'681/1'267'705 & \\
\hline & $\begin{array}{l}\text { ca. } 253,3(\mathrm{GW}- \\
\text { Mächtigkeit } \\
\text { ca. } 5 \mathrm{~m})\end{array}$ & $29 ’ 995$ & $\begin{array}{l}\text { Keine Messstelle GW- } \\
\text { Abstrom }\end{array}$ & \\
\hline & $\begin{array}{l}246,8 \text { (anste- } \\
\text { hender Fels bei } \\
248,2 \text { ) }\end{array}$ & & & \\
\hline \multirow{5}{*}{$\begin{array}{l}\text { 2: Tiefgarage } \\
\text { Clarastrasse } \\
\text { 2'611'855/1'267'951 }\end{array}$} & $-9,6$ & 957 & Waldshuterstrasse & \multirow{5}{*}{$\begin{array}{l}\text { Zentrale Lage Kleinbasler Innenstadt } \\
\text { Dauermietende, gewöhnlich wenig Verkehr }\end{array}$} \\
\hline & & & $(1064)$ & \\
\hline & 256,1 & $32 ’ 645$ & 2’613’221/1’268’751 & \\
\hline & 245,9 & 4’041 & Dolderweg (1161) & \\
\hline & $\begin{array}{l}244,8 \text { (oberhalb } \\
\text { anstehender Fels } \\
237,8)\end{array}$ & & 2’611’783/1’267’826 & \\
\hline \multirow{7}{*}{$\begin{array}{l}\text { 3: Autoeinstellhalle } \\
\text { St. Claraspital } \\
\text { 2'613'160/1'268'389 }\end{array}$} & $-8,5$ und $-11,2$ & 422 & $\begin{array}{l}\text { Waldshuterstrasse } \\
\text { (1064) }\end{array}$ & Tiefendifferenzierte Messung im 2. und im 3. UG \\
\hline & 259,9 & $34^{\prime} 653$ & $2^{\prime} 613^{\prime} 221 / 1^{\prime} 268^{\prime} 751$ & Hirzbrunnenquartier nahe Gemeindegrenze Riehen \\
\hline & 249,4 & 0 und 4'905 & $\begin{array}{l}\text { Wettsteinallee } 175 \\
(1075)\end{array}$ & $\begin{array}{l}89 \text { Parkplätze für Angestellte und Besuchende, un- } \\
\text { ter der Woche tagsüber sämtliche Plätze zwei bis } \\
\text { dreifach neu belegt, nachts steht es bis auf wenige } \\
\text { Dauermietplätze und Fahrzeuge von Nachtschichtan- } \\
\text { gestellten leer }\end{array}$ \\
\hline & 248,6 (oberhalb & & $2^{\prime} 612^{\prime} 875 / 1^{\prime} 267^{\prime} 963$ & \multirow{4}{*}{$\begin{array}{l}\text { Das unterste Stockwerk wird hauptsächlich vom } \\
\text { Personal genutzt, weshalb dort wochen- und } \\
\text { feiertags ebenfalls eine signifikant kleinere Anzahl } \\
\text { an Plätze belegt ist }\end{array}$} \\
\hline & $\begin{array}{l}\text { anstehender Fels } \\
241,3)\end{array}$ & & $\begin{array}{l}\text { Magdenweglein } 46 \\
\text { (3966) }\end{array}$ & \\
\hline & & & 2'613'161/1’268’064 & \\
\hline & & & $\begin{array}{l}\text { Zwei Messstellen } \\
\text { GW-Abstrom }\end{array}$ & \\
\hline \multirow{4}{*}{$\begin{array}{l}\text { 4: Tiefgarage St. } \\
\text { Jakob-Park } \\
\text { 2’613'763/1'265'596 }\end{array}$} & $-7,4$ & 8’094 & $\begin{array}{l}\text { G80-Karussell } \\
\text { (20J96) }\end{array}$ & \multirow{4}{*}{$\begin{array}{l}\text { Östliches Grossbasel, Kantonsgrenze zu Basel- } \\
\text { Landschaft, die streckenweise durch die Birs } \\
\text { gebildet wird } \\
680 \text { Parkplätze unterhalb des Fussballstadions stehen } \\
\text { tagsüber vor allem Kundschaft des Shoppingcenters } \\
\text { bzw. Gästen von Fussballspielen zur Verfügung, } \\
\text { nachts ist das Parkhaus geschlossen }\end{array}$} \\
\hline & 259,3 & $114 ’ 420$ & $2^{\prime} 613^{\prime} 376 / 1^{\prime} 264^{\prime} 591$ & \\
\hline & 255,1 & $59^{\prime} 802$ & Redingstrasse (749) & \\
\hline & $\begin{array}{l}250,3 \text { (anste- } \\
\text { hender Fels bei } \\
249,7 \text { ) }\end{array}$ & & 2’613’817/1’266’304 & \\
\hline \multirow{4}{*}{$\begin{array}{l}\text { 5: Parkhaus Anfos } \\
\text { 2'611'652/1'266' } 823\end{array}$} & $-16,0$ & $1 ’ 702$ & Heuwaage (931) & \multirow{4}{*}{$\begin{array}{l}\text { Grossbasler Innenstadt und dem Bahnhof SBB und } \\
\text { verfügt über } 166 \text { Stellplätze. Das unterste Stockwerk } \\
\text { steht Dauermietenden zur Verfügung, die oberen } \\
\text { tagsüber für Kurzzeitparking }\end{array}$} \\
\hline & 270,5 & $57^{\prime} 412$ & 2’611'226/1’266’714 & \\
\hline & $\begin{array}{l}257,6 \text { (Flurab- } \\
\text { stand ca. } 3,6 \mathrm{~m} \\
\text { Tiefe) }\end{array}$ & $12 ’ 155$ & Brunngässlein (3755) & \\
\hline & $\begin{array}{l}252,6 \text { (bis anste- } \\
\text { hender Fels bei } \\
254,0 \text { ) }\end{array}$ & & 2’611'754/1'266’922 & \\
\hline
\end{tabular}

GOK Geländeoberkante 


\section{Meteodaten}

Grundlage für die meteorischen Temperaturmessungen ( $\mathrm{T}_{\text {meteo }}$ ) lieferte die Meteodatenbank der Forschungsgruppe „Meteorology Climatology Remote Sensing (MCR)“ der Universität Basel. Für die zusammenhängenden Auswertungen mit den $\mathrm{T}_{\mathrm{TG}}$ dienten die Daten der beiden Messtationen BKLI und BAES (Abb. 1), welche laut MCR die repräsentativsten Referenztemperaturen für die Stadt Basel liefern, da sie den geringsten Interferenzen wie Strahlungseinflüssen oder fehlender Belüftung ausgesetzt sind. Die Messstation BKLI ist auf dem Dach des Geographie-Gebäudes der Universität Basel an der Klingelbergstrasse 27 installiert und zeichnet (u. a.) Temperaturdaten in $39 \mathrm{~m} \mathrm{Hö-}$ he auf, die Messstation BAES befindet sich auf dem Dach des Turmhauses am Aeschenplatz 2, Daten werden hier in $40 \mathrm{~m}$ Höhe erfasst (Feigenwinter et al. 2017). In dieser Arbeit wurden Stundenmittel-Messungen der beiden Standorte für den Messzeitraum der $\mathrm{T}_{\mathrm{TG}}$-Messungen verwendet, um potenzielle Korrelationen zwischen $\mathrm{T}_{\text {meteo }}$ und $\mathrm{T}_{\mathrm{TG}} \mathrm{zu}$ untersuchen.

\section{Grundwassertemperaturdaten}

Die Grundwasserstände und -temperaturen werden vom Amt für Umwelt und Energie, Kanton Basel-Stadt (AUE BS), durch ein Beobachtungsnetz von 85 GWM (davon fünf der Industriellen Werke Basel (IWB) und eine des Bundesamts für Umwelt (BAFU)) kontinuierlich überwacht und aufgezeichnet (AUE 2019). In dieser Arbeit wurden die Daten von acht GWM des AUE BS sowie ein Datensatz vom Amt für Umweltschutz und Energie, Kanton BaselLandschaft (AUE BL), verwendet (Tab. 1). Die Auswahl dieser neun GWM richtete sich primär nach der Orientierung der jeweiligen Standorte zur Position der Tiefgaragen im hydraulischen Gradienten (Abb. 1); bestenfalls konnte für jeden Tiefgaragenstandort mindestens eine GWM aufwärts des Grundwasserstromes sowie eine stromabwärts in die Datenauswertung einbezogen werden. Bedingt durch die Lage der ausgewählten Tiefgaragen war dies nicht für alle Standorte möglich; zwischen der Tiefgarage Storchen und dem Vorfluter (Rhein), in welchen das Grundwasser exfiltriert, existieren keine GWM. Für die Interpretation der Temperaturmessungen der Tunnelinnenluft des Autobahntunnels Nordtangente dienten die Daten der GWM SaintLouis-Strasse 2 (1443, Abb. 1).

\section{Statistische Datenverarbeitung}

Um die erfassten Messdaten in einen überschaubaren und interpretationsfähigen Rahmen zu bringen, wurden mit Microsoft Excel aus den CSV-Dateien bereinigte Datensätze als Ausgangsprodukt für weitere Bearbeitungen erstellt.
Die statistische Verarbeitung und Visualisierung der Daten erfolgte in der integrierten Entwicklungsumgebung RStudio mit der statistischen Programmiersprache $R$. Lineare Zusammenhänge von Temperaturschwankungen und lokalen Extrema der $\mathrm{T}_{\text {meteo }}$ und $\mathrm{T}_{\mathrm{TG}}$ konnten durch Analyse der Korrelationskoeffizienten $r$ nach Pearson berechnet werden, welcher definiert ist durch:

$r_{x, y}=\frac{\sum_{i=1}^{n}\left(x_{i}-\bar{x}\right)-\left(y_{i}-\bar{y}\right)}{\sqrt{\sum_{i=1}^{n}\left(x_{i}-\bar{x}\right)^{2}} * \sqrt{\sum_{i=1}^{n}\left(y_{i}-\bar{y}\right)^{2}}}$

mit $n$ dem Stichprobenumfang, $x_{i}$ und $y_{i}$ den Werten und den Mittelwerten $\bar{x}$ und $\bar{y}$ der Variablen $x$ und $y$. Der Pearson-Korrelationskoeffizient $\mathrm{r}$ nimmt einen Wert zwischen -1 und 1 an. Ein Wert kleiner 0 deutet auf eine negative Korrelation sowie einen linearen Zusammenhang höherer $\mathrm{x}$-Werte mit niedrigeren $\mathrm{y}$-Werten hin, Werte größer 0 deuten auf eine positive Korrelation sowie einen linearen Zusammenhang zwischen höheren Werten von $\mathrm{x}$ mit höheren Werten von y hin. Werte von $r \approx 0$ deuten auf einen fehlenden linearen Zusammenhang der $\mathrm{x}$ - und $\mathrm{y}$-Werte hin. Gerade bei Temperaturreihen kommt es vor, dass der Korrelationskoeffizient durch Trends und Saisonabhängigkeit verfälscht wird und vermeintlich höher ausfällt, als er eigentlich ist. Aus diesem Grund wurden die Korrelationskoeffizienten unter Einfluss verschiedener Faktoren getestet: Die unveränderten Datensätze, mit zeitlichem Versatz um Stunden, um eine allfällige verzögerte Reaktion der Temperaturschwankungen in den Tiefgaragen festzustellen, sowie das gleiche Verfahren mit Tagesmittelwerten und zeitlicher Verschiebung um Tage; die Datensätze, ihrer linearen Trends bereinigt, um die Beziehungen zeitlich kleinräumiger Temperaturschwankungen zwischen $\mathrm{T}_{\mathrm{TG}}$ und $\mathrm{T}_{\text {meteo }} \mathrm{zu}$ untersuchen, ebenfalls mit den gemessenen Stunden- und berechneten Tagesmitteltemperaturen. Zur „Enttrendung“ der Temperaturzeitreihen wurde für jeden Zeitpunkt ein Wert des linearen Gradienten extrahiert und von den Messtemperaturen desselben Zeitpunktes abgezogen.

\section{Resultate}

\section{Temperaturmessung Tiefgaragen}

Zur Verdeutlichung von Trends und Zusammenhängen in größerem Rahmen sind sowohl die tatsächlich gemessenen stündlichen Temperaturwerte als auch die gleitenden Mittelwerte von $\mathrm{T}_{\mathrm{TG}}$ und $\mathrm{T}_{\text {meteo }}$ dargestellt. Die Temperaturdaten der kantonalen GWM wurden, wenn möglich, in Abhängigkeit ihrer Position im Grundwasserzu- oder -abstrom zur jeweiligen $\mathrm{T}_{\mathrm{TG}}$-Messstelle abgebildet. Ebenfalls dargestellt sind die Resultate der $\mathrm{T}_{\mathrm{GW} \text { sim }}$ (Abb. 2). Die Beschreibung der Resultate bezieht sich auf den Beobachtungs- 

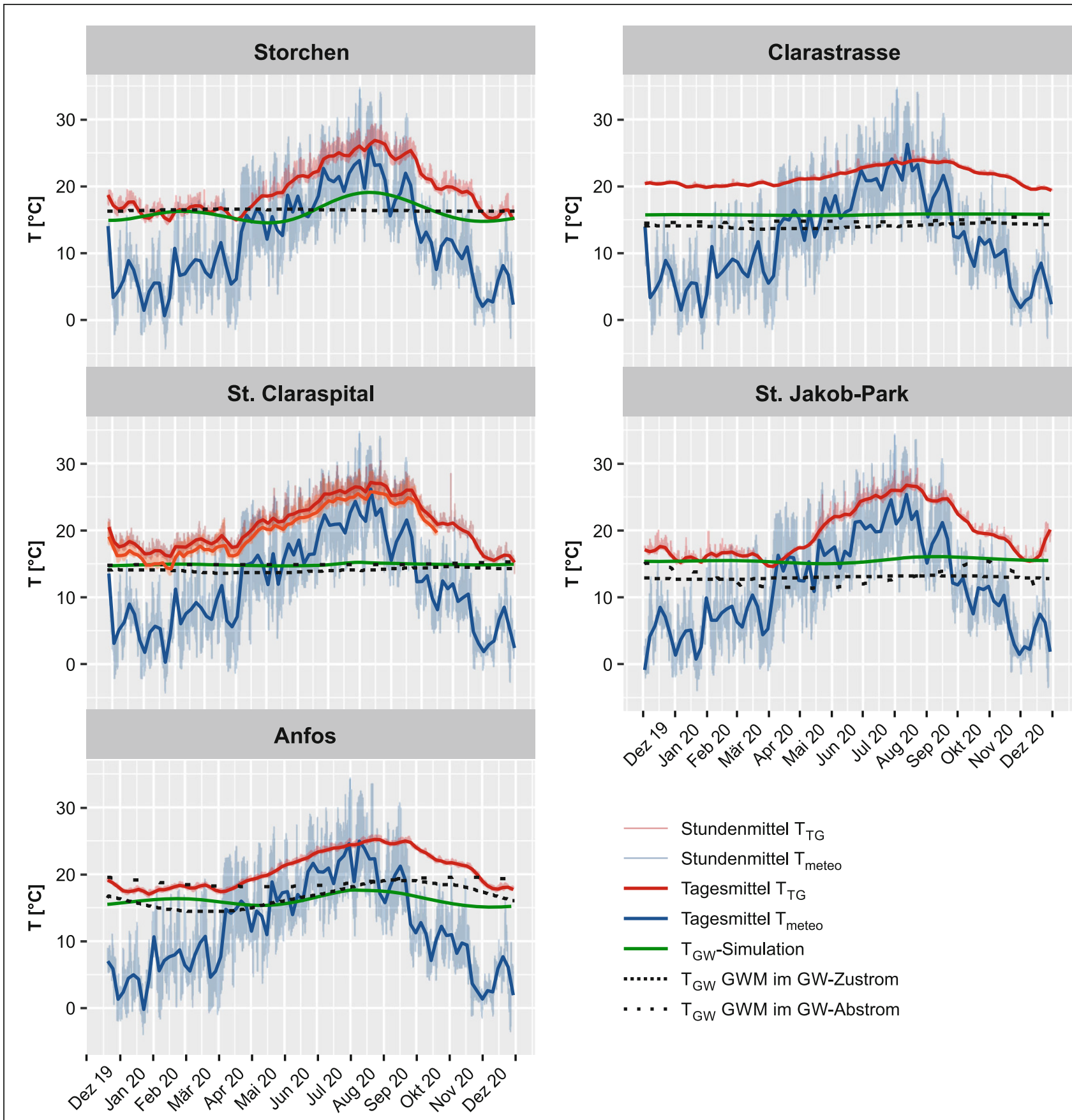

Abb. $2 \mathrm{~T}_{\mathrm{TG}}, \mathrm{T}_{\mathrm{GW}}, \mathrm{T}_{\mathrm{GW}}$ sim und $\mathrm{T}_{\text {meteo }}$ von Dezember 2019 bis Dezember 2020 für die Tiefgaragenstandorte (Standort St. Claraspital: dunkelrote Linie 2. UG, hellrote Linie 3. UG, $\mathrm{T}_{\mathrm{GW}}$ nur für Magdenweglein 46 (3966) dargestellt)

Fig. $2 \mathrm{~T}_{\mathrm{TG}}, \mathrm{T}_{\mathrm{GW}}, \mathrm{T}_{\mathrm{GW}}$ sim and $\mathrm{T}_{\text {meteo }}$ from December 2019 to December 2020 for the underground parkings (Location St. Claraspital: dark red line 2nd basement, light red line 3rd basement, $\mathrm{T}_{\mathrm{GW}}$ only shown for Magdenweglein 46 (3966))

zeitraum Dezember 2019 bis Dezember 2020. Genannte Jahreszeiten folgen der meteorologischen Einteilung und sind definiert als Winter $=$ Dezember-Februar (DJF), Frühling = März-Mai (MAM), Sommer $=$ Juni-August $($ JJA $)$ und Herbst $=$ September - November $(\mathrm{SON})$.

Die gemessenen $\mathrm{T}_{\mathrm{TG}}$ in der Tiefgarage Storchen (Abb. 2 und Tab. 2) resultieren in einem Mittelwert von $19,6^{\circ} \mathrm{C}$, wobei die gemessenen Temperaturen mit einer Abweichung von $1,4{ }^{\circ} \mathrm{C}$ um das gleitende Tagesmittel der Messreihe variieren. Das absolute Maximum liegt bei $29,4^{\circ} \mathrm{C}$ im August und das Minimum bei $14,2^{\circ} \mathrm{C}$ im Dezember. Die durchschnittliche $\Delta \mathrm{T}$ zu $\mathrm{T}_{\text {meteo }}$ (Mittelwert $12,2^{\circ} \mathrm{C}$ ) liegt bei $7,4^{\circ} \mathrm{C}$, wobei saisonale Unterschiede beobachtet werden können. Während die Abweichung im Winter 
Tab. 2 Resultate und statistische Daten der 5 Tiefgaragen-Messstandorte Table 2 Monitoring results and statistics of the 5 underground parkings

\begin{tabular}{|c|c|c|c|c|c|c|c|}
\hline Standort & $\begin{array}{l}{\overline{T_{T G}}}^{a} \\
{\left[{ }^{\circ} \mathrm{C}\right]} \\
\text { Jahr } \\
\text { Winter } \\
\text { Frühling } \\
\text { Sommer } \\
\text { Herbst }\end{array}$ & $\begin{array}{l}\overline{\Delta T_{\mathrm{TG}}{ }^{\mathrm{b}} \mathrm{b}} \\
{\left[{ }^{\circ} \mathrm{C}\right]}\end{array}$ & $\begin{array}{l}\overline{T_{\text {meteo }}} \mathrm{c} \\
{\left[{ }^{\circ} \mathrm{C}\right]}\end{array}$ & $\begin{array}{l}\overline{\Delta T_{\text {meteo }}}{ }^{\prime} \\
{\left[{ }^{\circ} \mathrm{C}\right]} \\
\text { Jahr } \\
\text { Winter } \\
\text { Frühling } \\
\text { Sommer } \\
\text { Herbst }\end{array}$ & $\begin{array}{l}\overline{T_{\mathrm{GW}} \uparrow \mathrm{e}} \\
{\left[{ }^{\circ} \mathrm{C}\right]} \\
\text { Messstation }\end{array}$ & $\begin{array}{l}\overline{T_{\mathrm{GW}} \downarrow^{\mathrm{f}}} \\
{\left[{ }^{\circ} \mathrm{C}\right]}\end{array}$ & $\begin{array}{l}\overline{T_{\mathrm{GWsim}}}{ }^{\mathrm{g}} \\
{\left[{ }^{\circ} \mathrm{C}\right]}\end{array}$ \\
\hline $\begin{array}{l}\text { 1: Stor- } \\
\text { chen }\end{array}$ & $\begin{array}{l}19,6 \\
16,5 \\
17,6 \\
23,3 \\
21,0\end{array}$ & 1,4 & 12,2 & $\begin{array}{l}7,4 \\
10,4 \\
5,2 \\
3,8 \\
8,9\end{array}$ & $\begin{array}{l}16,4 \\
1052\end{array}$ & - & 16,2 \\
\hline $\begin{array}{l}2: \\
\text { Clara- } \\
\text { strasse }\end{array}$ & $\begin{array}{l}21,5 \\
20,2 \\
20,8 \\
22,8 \\
22,4\end{array}$ & 0,2 & 12,2 & $\begin{array}{l}9,1 \\
14,2 \\
8,4 \\
2,7 \\
10,3\end{array}$ & $\begin{array}{l}14,1 \\
1064\end{array}$ & $\begin{array}{l}14,8 \\
1161\end{array}$ & 15,8 \\
\hline $\begin{array}{l}\text { 3: St. } \\
\text { Clara- } \\
\text { spital, } \\
\text { 2. UG }\end{array}$ & $\begin{array}{l}20,8 \\
17,6 \\
20,0 \\
25,0 \\
22,0\end{array}$ & 1,3 & 12,2 & $\begin{array}{l}8,4 \\
11,5 \\
7,6 \\
5,0 \\
9,9\end{array}$ & $\begin{array}{l}14,1 \\
1064\end{array}$ & $\begin{array}{l}14,9 \\
3966 \\
14,8 \\
1075\end{array}$ & 14,8 \\
\hline $\begin{array}{l}\text { 3: St. } \\
\text { Clara- } \\
\text { spital, } \\
\text { 3. UG }\end{array}$ & $\begin{array}{l}19,6 \\
16,1 \\
18,9 \\
23,8 \\
20,8\end{array}$ & 1,5 & & $\begin{array}{l}7,1 \\
10,1 \\
6,5 \\
3,7 \\
8,7\end{array}$ & & & 14,9 \\
\hline $\begin{array}{l}\text { 4: St. } \\
\text { Jakob- } \\
\text { Park }\end{array}$ & $\begin{array}{l}19,6 \\
16,4 \\
17,1 \\
24,1 \\
21,0\end{array}$ & 1,2 & 11,8 & $\begin{array}{l}7,0 \\
10,9 \\
5,1 \\
4,4 \\
9,3\end{array}$ & $\begin{array}{l}13,0 \\
20 J 96\end{array}$ & $\begin{array}{l}12,9 \\
749\end{array}$ & 15,3 \\
\hline $\begin{array}{l}\text { 5: An- } \\
\text { fos }\end{array}$ & $\begin{array}{l}20,7 \\
17,9 \\
18,8 \\
23,2 \\
22,8\end{array}$ & 0,3 & 11,8 & $\begin{array}{l}8,9 \\
12,3 \\
6,8 \\
3,4 \\
11,1\end{array}$ & $\begin{array}{l}16,7 \\
931\end{array}$ & $\begin{array}{l}18,9 \\
3755\end{array}$ & 16,1 \\
\hline
\end{tabular}

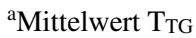

${ }^{\mathrm{b}}$ Durchschnittliche Abweichung $\mathrm{T}_{\mathrm{TG}}$ zum gleitenden $\mathrm{T}_{\mathrm{TG}}$-Tagesmittel

${ }^{c}$ Mittelwert $\mathrm{T}_{\text {meteo }}$

${ }^{\mathrm{d}}$ Durchschnittliche Abweichung $\mathrm{T}_{\mathrm{TG}} \mathrm{zu} \mathrm{T}_{\text {meteo }}$

${ }^{\mathrm{e}}$ Mittelwert $\mathrm{T}_{\mathrm{GW}}$ Grundwasserzustrom

${ }^{\mathrm{f}}$ Mittelwert $\mathrm{T}_{\mathrm{GW}}$ Grundwasserabstrom

${ }^{\mathrm{g}}$ Mittelwert $\mathrm{T}_{\mathrm{GW}} \mathrm{sim}$

durchschnittlich $10,4^{\circ} \mathrm{C}$ beträgt, sind es im Frühling nur $5,2^{\circ} \mathrm{C}$, in den Sommermonaten nur noch $3,8^{\circ} \mathrm{C}$ und im Herbst wieder $8,9^{\circ} \mathrm{C}$. $\mathrm{T}_{\mathrm{GW}}$ an der GWM Bernoullianum (1052), welche sich $460 \mathrm{~m}$ nordwestlich und im Grundwasserzustrom der Tiefgarage befindet (Abb. 1 und Tab. 1), zeigt lediglich geringe Temperaturschwankungen zwischen 16,3 und $16,6^{\circ} \mathrm{C}$, mit einem Mittelwert von $16,4^{\circ} \mathrm{C}$. $\mathrm{T}_{\mathrm{GW}}-$ Maxima werden zwischen April und Juni erreicht, Minima im Dezember. Für $\mathrm{T}_{\mathrm{GWsim}}$ resultierten ähnliche Werte, der Mittelwert beträgt $16,2^{\circ} \mathrm{C}$, allerdings zeigt die Temperatur hier deutliche saisonale Schwankungen mit einem lokalen Maximum um $16,3^{\circ} \mathrm{C}$ im Winter und einem Minimum um $14,5^{\circ} \mathrm{C}$ zu Beginn des Sommers bei anschließend starkem Anstieg bis auf $19,1^{\circ} \mathrm{C}$ in den Sommermonaten.

Mit $21,5^{\circ} \mathrm{C}$ wird an der Clarastrasse der höchste $\mathrm{T}_{\mathrm{TG}^{-}}$ Mittelwert gemessen, wobei hier auch die geringsten täglichen sowie gesamtzeitlichen Schwankungen zu beobachten sind (Abb. 2 und Tab. 2). Das absolute Maximum liegt bei $25,3{ }^{\circ} \mathrm{C}$ im August und das Minimum bei $19,4{ }^{\circ} \mathrm{C}$ im Dezember. Die durchschnittliche $\Delta \mathrm{T}$ zu $\mathrm{T}_{\text {meteo }}$ (Mittelwert $12,2^{\circ} \mathrm{C}$ ) liegt bei $9,3^{\circ} \mathrm{C}$. Dabei betragen die durchschnittlichen Abweichungen vom gleitenden $\mathrm{T}_{\mathrm{TG}}$-Mittelwert $0,2^{\circ} \mathrm{C}$. Die geringere Temperaturschwankung im Jahresverlauf bewirkt große bzw. kleine jahreszeitliche $\Delta \mathrm{T}$ zwischen $\mathrm{T}_{\text {meteo }}$ und $\mathrm{T}_{\mathrm{TG}}$ von 10,3 im Herbst, 14,2 im Winter über 8,4 im 
Frühling bis $\mathrm{zu} 2,7^{\circ} \mathrm{C}$ in den Sommermonaten. Die beiden GWM befinden sich $1,6 \mathrm{~km}$ nordöstlich (Waldshuterstrasse 1064) und $140 \mathrm{~m}$ südwestlich (Dolderweg 1161) im Grundwasserzu- und -abstrom der Tiefgaragen (Abb. 1 und Tab. 1). Auch diese beiden Messstationen weisen keine großen Temperaturschwankungen des Grundwassers im Jahresverlauf auf. Bei GWM 1064 variiert das $\mathrm{T}_{\mathrm{GW}}$-Tagesmittel zwischen 14,4 im Dezember und $13,6^{\circ} \mathrm{C}$ im März mit einem Gesamtmittel von $14,1^{\circ} \mathrm{C}$. Sowohl im Winter als auch im Sommer werden höhere Temperaturen als im Frühling verzeichnet. Bei GWM 1161 schwankt das $\mathrm{T}_{\mathrm{GW}}$-Tagesmittel zwischen 14,5 und $15,0^{\circ} \mathrm{C}$, ein klarer Trend über die Zeit ist hier nicht erkennbar. Der Mittelwert von $14,8^{\circ} \mathrm{C}$ ist an dieser GWM um $0,7^{\circ} \mathrm{C}$ höher als jener bei GWM 1064. $\mathrm{T}_{\mathrm{GWsim}}$ beträgt an diesem Standort $15,8^{\circ} \mathrm{C}$ und zeigt keine großen Abweichungen.

In der Tiefgarage St. Claraspital wurden auf zwei Stockwerken $\mathrm{T}_{\mathrm{TG}}$-Messungen durchgeführt (2. UG und 3. UG; Abb. 2 und Tab. 2; aufgrund einer technischen Fehlfunktion wurden aus dem 3. UG nur Daten bis Mitte Oktober erfasst). Beide Temperaturkurven zeigen einen sehr gleichförmigen Verlauf, mit einer konstanten $\Delta \mathrm{T}$ von ca. $1,2^{\circ} \mathrm{C}$. Die mittleren Temperaturen betragen hier $20,8{ }^{\circ} \mathrm{C}$ im 2 . UG und $19,6^{\circ} \mathrm{C}$ im 3 . UG und sind damit um 8,6 bzw. $7,4^{\circ} \mathrm{C}$ höher als der $\mathrm{T}_{\text {meteo-Mittelwert }}\left(12,2^{\circ} \mathrm{C}\right)$. Die Abweichungen vom gleitenden Tagesmittel fielen im höher gelegenen 2 . UG etwas kleiner aus, durchschnittlich betrugen sie hier $1,3^{\circ} \mathrm{C}$, im 3. UG waren es rund $1,5^{\circ} \mathrm{C}$. Der Wertebereich der $\mathrm{T}_{\mathrm{TG}^{-}}$ Tagesmittel liegt zwischen 30,4 und $13,5^{\circ} \mathrm{C}$ im 2 . UG bzw. zwischen 29,0 und $13,4^{\circ} \mathrm{C}$ im 3 . UG. Dies sind sowohl die höchsten als auch die tiefsten gemessenen Tagesmitteltemperaturen aller Standorte. Für den Standort St. Claraspital stehen die Datensätze von drei GWM zur Verfügung. Die GWM Waldshuterstrasse (1064) liegt $370 \mathrm{~m}$ nördlich im Grundwasserzustrom der Tiefgarage. Die GWM Magdenweglein 46 (3966) liegt $320 \mathrm{~m}$ südlich und die GWM Wettsteinallee 175 (1075) 500m südwestlich, beide im Grundwasserabstrom der Tiefgarage (Abb. 1 und Tab. 1). Die $\mathrm{T}_{\mathrm{GW}^{-}}$ Mittelwerte der Messstation südlich der Tiefgarage liegen mit $14,9^{\circ} \mathrm{C}$ bei GWM 3966 und $14,8^{\circ} \mathrm{C}$ bei GWM 1075 nah beieinander, wobei letztere in den Sommermonaten Minima von $14,5^{\circ} \mathrm{C}$ erreicht, während die Temperaturabweichungen an GWM 3966 über den gesamten Verlauf der Aufzeichnung $0,1^{\circ} \mathrm{C}$ nicht über- bzw. unterschreiten. Die $\mathrm{T}_{\mathrm{GW}}$ bei GWM 1064 zeigt, wie bereits für den Standort der Tiefgarage Clarastrasse beschrieben, einen Temperaturverlauf mit Minima von $13,6^{\circ} \mathrm{C}$ im Frühling. Mit einem Mittelwert von $14,1^{\circ} \mathrm{C}$ liegt die Temperatur hier rund $1^{\circ} \mathrm{C}$ unter den Mittelwerten der anderen beiden GWM. Für $\mathrm{T}_{\mathrm{GWsim}}$ wurden die Simulationsergebnisse für die jeweiligen Messhöhen der beiden $\mathrm{T}_{\mathrm{TG}}-$ Messungen extrahiert, sie liegen bei $14,9^{\circ} \mathrm{C}$ in der grundwassergesättigten und bei $14,8^{\circ} \mathrm{C}$ in der grundwasserungesättigten Zone.
Die $\mathrm{T}_{\mathrm{TG}}$-Messung des Standorts St. Jakob-Park zeigt einen ähnlichen Verlauf wie die Messungen in der Tiefgarage des St. Claraspitals (Abb. 2 und Tab. 2). Der Mittelwert beträgt $19,6^{\circ} \mathrm{C}, 7,8^{\circ} \mathrm{C}$ höher als der $\mathrm{T}_{\text {meteo- }}$ Mittelwert $\left(11,8^{\circ} \mathrm{C}\right)$, das $\mathrm{T}_{\mathrm{TG}}$-Tagesmittel bewegt sich zwischen $14,5{ }^{\circ} \mathrm{C}$ im Winter und $27,2^{\circ} \mathrm{C}$ im Sommer. Die Abweichungen vom gleitenden Tagesmittel betragen durchschnittlich $1,2^{\circ} \mathrm{C}$. Die GWM G80 Karussell (20J96) befindet sich $1,1 \mathrm{~km}$ südsüdöstlich im Grundwasserzustrom der Tiefgarage St. Jakob-Park (Abb. 1). Der hier gemessene Mittelwert beträgt $13,0^{\circ} \mathrm{C}$, mit absoluten Minima von $12,6^{\circ} \mathrm{C}$ im März und Maxima von $13,3^{\circ} \mathrm{C}$ im September. Die mittlere $\Delta \mathrm{T}$ zwischen $\mathrm{T}_{\mathrm{TG}}$ und $\mathrm{T}_{\mathrm{GW}}$ beträgt im Winter 3,4, im Frühling 4,1, im Sommer 11,1 und im Herbst $8,0^{\circ} \mathrm{C}$. Die zweite GWM (Redingstrasse 749) liegt $710 \mathrm{~m}$ nördlich im Grundwasserabstrom der Tiefgarage St. JakobPark (Abb. 1 und Tab. 1). Der $\mathrm{T}_{\mathrm{GW}}$-Mittelwert beträgt hier $12,9^{\circ} \mathrm{C}, 0,1^{\circ} \mathrm{C}$ niedriger im Vergleich zu GWM 20J96. Die $\mathrm{T}_{\mathrm{GW}}$ zeigen hier allerdings eine deutlich stärkere Schwankung über die Zeit, mit einem Maximum von $15,1^{\circ} \mathrm{C}$ im Dezember und einem Minimum von $11,3^{\circ} \mathrm{C}$ im Mai, liegen also zu Beginn der Messperiode um $2{ }^{\circ} \mathrm{C}$ höher als die $\mathrm{T}_{\mathrm{GW}}$ bei GWM 20J96, unterschreiten diese allerdings im Februar, nähern sich ihnen ab Mai wieder an und überschreiten sie wiederum im August. Der Mittelwert der $\mathrm{T}_{\mathrm{GWsim}}$ des Standorts St. Jakob-Park beträgt $15,3{ }^{\circ} \mathrm{C}$, wobei der Verlauf von $\mathrm{T}_{\mathrm{GW}}$ sim nur sehr schwache Temperaturschwankungen zeigt.

Der $\mathrm{T}_{\mathrm{TG}}$-Mittelwert am Standort Anfos beträgt $20,7^{\circ} \mathrm{C}$,

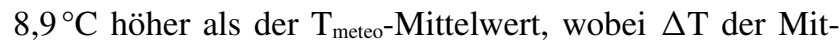
telwerte saisonalen Schwankungen ausgesetzt ist, von 12,3 im Winter über $6,8^{\circ} \mathrm{C}$ im Frühling zu $3,4{ }^{\circ} \mathrm{C}$ im Sommer zu $11,1^{\circ} \mathrm{C}$ im Herbst (Abb. 2 und Tab. 2). Die Temperaturschwankungen um das gleitende $\mathrm{T}_{\mathrm{TG}}$-Tagesmittel betragen im Schnitt $1,1^{\circ} \mathrm{C}$. Auch für diesen Standort stehen die Daten von zwei GWM zur Verfügung: Die GWM Heuwaage (931) befindet sich ca. $430 \mathrm{~m}$ westlich im Grundwasserzustrom der Tiefgarage. Die mittlere $\mathrm{T}_{\mathrm{GW}}$ beträgt hier $16,7^{\circ} \mathrm{C}$, mit einem Maximum von $19,2^{\circ} \mathrm{C}$ im September und einem Minimum von $14,5^{\circ} \mathrm{C}$ im März. $\Delta \mathrm{T}$ zwischen $\mathrm{T}_{\mathrm{GW}}$ und $\mathrm{T}_{\mathrm{TG}}$ beträgt $4,0^{\circ} \mathrm{C}$. Die zweite GWM Brunngässlein (3755) liegt ca. $170 \mathrm{~m}$ nordöstlich im Grundwasserabstrom der Tiefgarage (Abb. 1 und Tab. 1). Der $\mathrm{T}_{\mathrm{TG}}$-Mittelwert liegt hier bei $18,9^{\circ} \mathrm{C}$ mit einem Maximum von $19,7^{\circ} \mathrm{C}$ im November und einem Minimum von $18,2^{\circ} \mathrm{C}$ im Mai. Die Temperatur ist hier durchschnittlich $2,2^{\circ} \mathrm{C}$ höher als an der GWM 931,

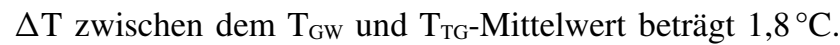
Die $\mathrm{T}_{\mathrm{GWsim}}$ resultiert am Standort Anfos in einem Mittelwert von $16,1^{\circ} \mathrm{C}$; auch hier ist, ähnlich wie für die Tiefgarage Storchen, ein Temperaturjahresgang zu erkennen, mit einem lokalen Maximum von $16,4^{\circ} \mathrm{C}$ zum Ende des Winters und einem lokalen Minimum von $15,4^{\circ} \mathrm{C}$ zum Sommerbeginn. 
Tab. 3 Pearson-Korrelationskoeffizienten $\mathrm{r}$ zwischen Stundenwerten von $\mathrm{T}_{\mathrm{TG}}$ und $\mathrm{T}_{\text {meteo, }}$, vor und nach extrahieren des Trends. Pearson-Korrelationskoeffizienten $\mathrm{r}$ zwischen trendbereinigten Stundenwerten und Tagesmittelwerten von $\mathrm{T}_{\mathrm{TG}}$ und $\mathrm{T}_{\text {meteo }}$ mit zeitlichen Versätzen von $\mathrm{T}_{\mathrm{TG}}$ in $24 \mathrm{~h}$ Schritten; Höchstwerte kursiv hervorgehoben

Table 3 Pearson-correlation-coefficient $\mathrm{r}$ between hourly values of $\mathrm{T}_{\mathrm{TG}}$ and $\mathrm{T}_{\mathrm{meteo}}$, before and after extracting the trends. Pearson-correlationcoefficient $r$ between trend-adjusted hourly values and daily mean values of $\mathrm{T}_{\mathrm{TG}}$ and $\mathrm{T}_{\text {meteo }}$ with time offsets of $\mathrm{T}_{\mathrm{TG}}$ in $24 \mathrm{~h}$ steps; maximum values highlighted italic

\begin{tabular}{|c|c|c|c|c|c|c|c|c|c|}
\hline Standort & $\begin{array}{l}\text { Trend vor- } \\
\text { handen }\end{array}$ & $\begin{array}{l}\text { Trend } \\
\text { entfernt }\end{array}$ & $\begin{array}{l}\text { Zeitliche Auflö- } \\
\text { sung }\end{array}$ & 0 & $+24 \mathrm{~h}$ & $+48 \mathrm{~h}$ & $+72 \mathrm{~h}$ & $+96 \mathrm{~h}$ & $+120 \mathrm{~h}$ \\
\hline 1: Storchen & 0,81 & 0,47 & $\begin{array}{l}\text { Stundenwerte } \\
\text { Tagesmittel }\end{array}$ & $\begin{array}{l}0,44 \\
0,48\end{array}$ & $\begin{array}{l}0,47 \\
0,52\end{array}$ & $\begin{array}{l}0,46 \\
0,51\end{array}$ & $\begin{array}{l}0,44 \\
0,48\end{array}$ & $\begin{array}{l}0,42 \\
0,45\end{array}$ & $\begin{array}{l}0,69 \\
0,42\end{array}$ \\
\hline 2: Clarastrasse & 0,78 & 0,36 & $\begin{array}{l}\text { Stundenwerte } \\
\text { Tagesmittel }\end{array}$ & $\begin{array}{l}0,29 \\
0,37\end{array}$ & $\begin{array}{l}0,34 \\
0,42\end{array}$ & $\begin{array}{l}0,36 \\
0,45\end{array}$ & $\begin{array}{l}0,36 \\
0,45\end{array}$ & $\begin{array}{l}0,35 \\
0,44\end{array}$ & $\begin{array}{l}0,34 \\
0,43\end{array}$ \\
\hline $\begin{array}{l}\text { 3: St. Claraspital, } \\
\text { 2. UG }\end{array}$ & 0,86 & 0,57 & $\begin{array}{l}\text { Stundenwerte } \\
\text { Tagesmittel }\end{array}$ & $\begin{array}{l}0,56 \\
0,57\end{array}$ & $\begin{array}{l}0,57 \\
0,60\end{array}$ & $\begin{array}{l}0,54 \\
0,55\end{array}$ & $\begin{array}{l}0,51 \\
0,52\end{array}$ & $\begin{array}{l}0,49 \\
0,49\end{array}$ & $\begin{array}{l}0,44 \\
0,43\end{array}$ \\
\hline $\begin{array}{l}\text { 3: St. Claraspital, } \\
\text { 3. UG }\end{array}$ & 0,85 & 0,53 & $\begin{array}{l}\text { Stundenwerte } \\
\text { Tagesmittel }\end{array}$ & $\begin{array}{l}0,45 \\
0,46\end{array}$ & $\begin{array}{l}0,52 \\
0,56\end{array}$ & $\begin{array}{l}0,53 \\
0,57\end{array}$ & $\begin{array}{l}0,52 \\
0,56\end{array}$ & $\begin{array}{l}0,52 \\
0,55\end{array}$ & $\begin{array}{l}0,47 \\
0,49\end{array}$ \\
\hline 4: St. Jakob-Park & 0,75 & 0,32 & $\begin{array}{l}\text { Stundenwerte } \\
\text { Tagesmittel }\end{array}$ & $\begin{array}{l}0,29 \\
0,31\end{array}$ & $\begin{array}{l}0,32 \\
0,35\end{array}$ & $\begin{array}{l}0,31 \\
0,34\end{array}$ & $\begin{array}{l}0,31 \\
0,33\end{array}$ & $\begin{array}{l}0,30 \\
0,32\end{array}$ & $\begin{array}{l}0,29 \\
0,31\end{array}$ \\
\hline 5: Anfos & 0,77 & 0,22 & $\begin{array}{l}\text { Stundenwerte } \\
\text { Tagesmittel }\end{array}$ & $\begin{array}{l}0,15 \\
0,15\end{array}$ & $\begin{array}{l}0,19 \\
0,20\end{array}$ & $\begin{array}{l}0,20 \\
0,22\end{array}$ & $\begin{array}{l}0,21 \\
0,23\end{array}$ & $\begin{array}{l}0,22 \\
0,23\end{array}$ & $\begin{array}{l}0,20 \\
0,22\end{array}$ \\
\hline
\end{tabular}

\section{Korrelationen}

Tab. 3 zeigt die Pearson-Korrelationskoeffizienten $r$ der stündlichen Messungen von $\mathrm{T}_{\mathrm{TG}}$ und $\mathrm{T}_{\text {meteo }}$. Die Korrelationsanalyse wurde für jeden Standort einmal vor und ein weiteres Mal nach dem Entfernen des linearen Trends, der durch den Temperaturanstieg über die Frühlings- und Sommermonate bedingt ist, durchgeführt. Die unveränderten Temperaturreihen weisen eine starke positive Korrelation mit Koeffizienten zwischen 0,86 und 0,75 auf. Nach Bereinigung des Trends resultiert der Korrelationskoeffizient in tieferen Werten zwischen 0,57 und 0,22, bleibt aber weiterhin im positiven Bereich. Für die weitere statistische Analyse wurden die durch den Trend unbeeinflussten Daten verwendet.

Tab. 3 zeigt auch die Resultate der Pearson-Korrelationsanalyse der trendbereinigten Temperaturreihen. Dabei wurden hier sowohl die Korrelationen der stündlichen Messwerte sowie die der diskreten Tagesmittelwerte verglichen. Zur Untersuchung, ob in den Tiefgaragen eine verzögerte Reaktion auf Temperaturschwankungen der atmosphärischen Temperaturen besteht, wurde die Analyse jedes Standortes wiederholt durchgeführt, bis eine maximale Korrelation erreicht wurde. Aus Tab. 3 geht hervor, dass eine solche Verzögerung existiert und, je nach Standort, zwischen einem und vier Tagen beträgt. Im Vergleich zwischen Stundenund Tagesmittelwerten fallen die Korrelationskoeffizienten der Tagesmittel für jeden Standort höher aus als jene der stündlichen Messungen.

\section{Temperaturmessung Nordtangente}

Die Mittelwerte der gemessenen Temperaturen im Tunnelbauwerk Nordtangente liegen zwischen $13,7{ }^{\circ} \mathrm{C}$ (Tunnel- portal) und $18,1^{\circ} \mathrm{C}$ (Abfahrt TLR), die tiefsten Temperaturmittelwerte wurden also in der Nähe der Einfahrt, die höchsten im Tunnelinneren gemessen. Die tiefsten Messwerte betragen $-0,3$ bis $7,7^{\circ} \mathrm{C}$, die Maxima liegen zwischen 28,4 und $32,8^{\circ} \mathrm{C}$. So entstehen sowohl in den Winterals auch in den Sommermonaten Unterschiede zur Grundwassertemperatur von bis $\mathrm{zu}-15,3$ bzw. $17,8^{\circ} \mathrm{C}$. Die Temperaturmessungen bilden im Jahresgang eine sinusförmige Kurve, die in etwa um die mittlere $\mathrm{T}_{\mathrm{GW}}$ von ca. $15^{\circ} \mathrm{C}$ oszilliert, mit einem Minimum im Winter, einem Maximum im Sommer und Schnittpunkten mit $\mathrm{T}_{\mathrm{GW}}$ in den Frühlingsund Herbstmonaten (Abb. 3).

\section{Diskussion}

\section{Korrelationen und Temperaturtagesgang}

Die Resultate der ersten Korrelationsanalyse (Tab. 3) deuten darauf hin, dass die Schwankungen von $\mathrm{T}_{\mathrm{TG}}$ sowohl dem Jahresverlauf als auch kurzfristigen Veränderungen von $\mathrm{T}_{\text {meteo }}$ folgen. Auf den ersten Blick spiegeln sich diese Schwankungen deutlich in den stündlichen Messungen der Tiefgaragen Storchen, St. Claraspital und St. JakobPark wider, mit täglicher $\Delta \mathrm{T}$ von mehr als $2^{\circ} \mathrm{C}$ vom Tagesmittelwert. Die tägliche $\Delta \mathrm{T}$ der anderen beiden Standorte fallen geringer aus; in der Tiefgarage Anfos betragen diese $1,1^{\circ} \mathrm{C}$ und am Standort Clarastrasse nur $0,1^{\circ} \mathrm{C}$. $\mathrm{T}_{\mathrm{TG}}$ der Standorte St. Claraspital, Storchen und St. Jakob-Park weisen die größten Standardabweichungen auf (Tab. 4 und Abb. 4).

Dass die täglichen Temperaturschwankungen nicht von der Standorttiefe abhängig sind, zeigen u.a. die Werte der Messung im 3. UG der Tiefgarage des St. Claraspitals. 
Abb. 3 Gemessene Temperaturtagesmittel der Tunnelinnenluft an verschiedenen Standorten des Tunnelbauwerkes Nordtangente; dargestellt zusammen mit mittlerer Temperatur an der Grundwassermessstation SaintLouis-Strasse 2 (1443, Abb. 1) Fig. 3 Measured daily averages of the tunnel air temperature at different locations of the freeway tunnel Nordtangente; shown together with mean temperature at the groundwater observation well Saint-Louis-Strasse 2 (1443, Abb. 1)

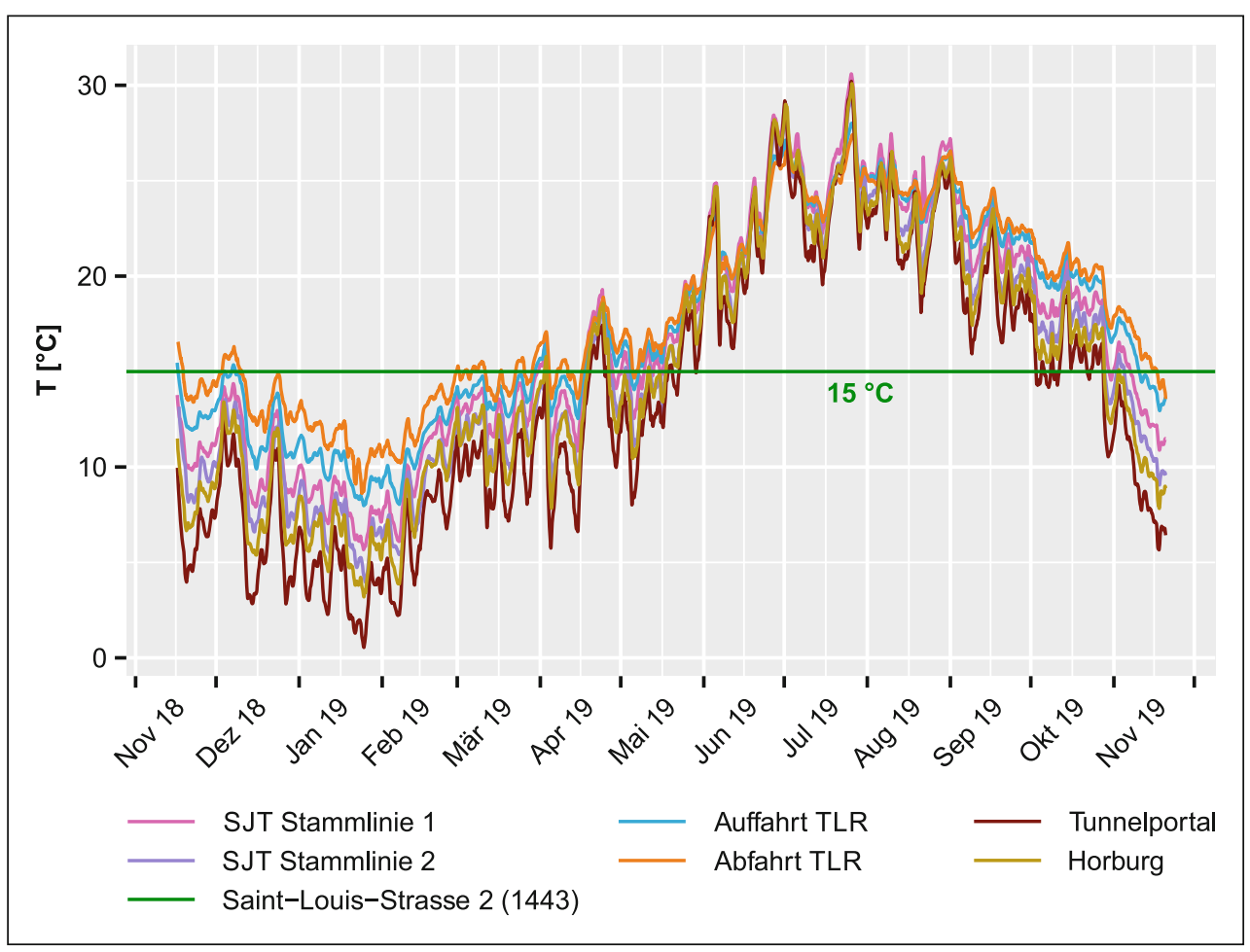

Der mit 11,2m Distanz zur Geländeoberkante zweittiefste Standort zeigt auch die zweithöchsten täglichen Temperaturschwankungen nach der Messung im 3. UG der Tiefgarage. Es muss also einen anderen Faktor als $\mathrm{T}_{\text {meteo }}$ geben, welcher die täglichen $\mathrm{T}_{\mathrm{TG}}$-Ausschläge begründet und dazu führt, dass die Korrelationskoeffizienten der Stundenwerte tiefer ausfallen als jene der Tagesmittelwerte. Deshalb wurde eine genauere Analyse der Temperaturverläufe einzelner Wochen an den Standorten Storchen, St. Claraspital und St. Jakob-Park vorgenommen. Es wird deutlich,

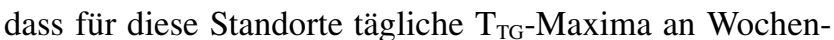
enden und Feiertagen fehlen bzw. schwächer ausgeprägt sind als jene an den Standorten Clarastrasse und Anfos. Dieses Phänomen zeigt sich besonders deutlich im Monat März. Aufgrund der COVID-19-Pandemie wurde am 16. März 2020 in der Schweiz die außerordentliche Lage gemäß Epidemiegesetz erklärt. Diese hatte zur Folge, dass, laut Medienmitteilung des Bundesamtes für Gesundheit (BAG) vom 13. März 2020, ,alle Läden, Märkte, Restaurants, Bars sowie Freizeit- und Unterhaltungsbetriebe“ zwischen dem 16. März und dem 11. Mai 2020 geschlossen wurden (BAG 2020a). In diesem Zeitraum fallen die $\mathrm{T}_{\mathrm{TG}^{-}}$ Schwankungen kleiner aus, im Vergleich zum $\mathrm{T}_{\text {meteo-Tages- }}$ gang, der mehr oder weniger unverändert bleibt. Der größte beeinflussende Faktor ist dementsprechend die Nutzungsart der Tiefgaragen, also die Anzahl der ein- und ausfahrenden Fahrzeuge und der Betrieb von Kühl-, bzw. Heizanlagen, welche an Wochenenden und Feiertagen sowie während des „Lockdowns“ im Frühjahr deutlich geringer ausfallen. Am
18. Dezember 2020 erließ der Bundesrat eine erneute Verordnung zur Schließung von Restaurants, Freizeit-, Sport und Kultureinrichtungen ab dem 22. Dezember 2020 (BAG 2020b). Einkaufsläden und einige Dienstleistungsbetriebe waren von den Maßnahmen nicht betroffen, demzufolge dieser zweite „Lockdown“ auch nur eine geringfügige Änderung der Tiefgaragennutzung bewirkte. Abb. 5 zeigt die täglichen Temperaturgänge in den Tiefgaragen, dargestellt in Kalenderwochen. In der Darstellung farblich hervorgehoben sind einige Wochen, die eine oder mehrere Anomalien wie z. B. Feiertage enthalten; konkret sind das einer der verkaufsoffenen Sonntage in der Adventszeit am 15.12.2019, die Weihnachtsfeiertage vom 25. bis 26.12.2019, Neujahr am 1. Januar 2020, eine Woche der durch den „Lockdown“ verordneten Betriebsschließungen vom 23. bis 29.03.2020, Christi Himmelfahrt am 21.05.2020, der Pfingstmontag am 01.06.2020 und der schweizerische Nationalfeiertag am 01.08.2020. Wie erwartet sind in den verschiedenen Tiefgaragen unterschiedliche Reaktionen zu beobachten.

Für die Tiefgarage Storchen spiegelt sich die vorwiegend von Kunden der umliegenden Läden und Geschäfte geprägte Nutzung im Verlauf von $\mathrm{T}_{\mathrm{TG}}$ wider (Abb. 5). Obwohl die Tiefgarage an 365 Tagen im Jahr geöffnet ist, bleiben Temperaturschwankungen an allen gesetzlichen Feiertagen aus. Am verkaufsoffenen Sonntag ist hingegen eine deutlich höhere Temperatur zu verzeichnen als an den restlichen Tagen dieser Woche. Auch im Vergleich mit anderen Sonntagen ist der Unterschied signifikant. In der ausgewählten Woche während des „Lockdowns“ bleiben die sonst kenn- 


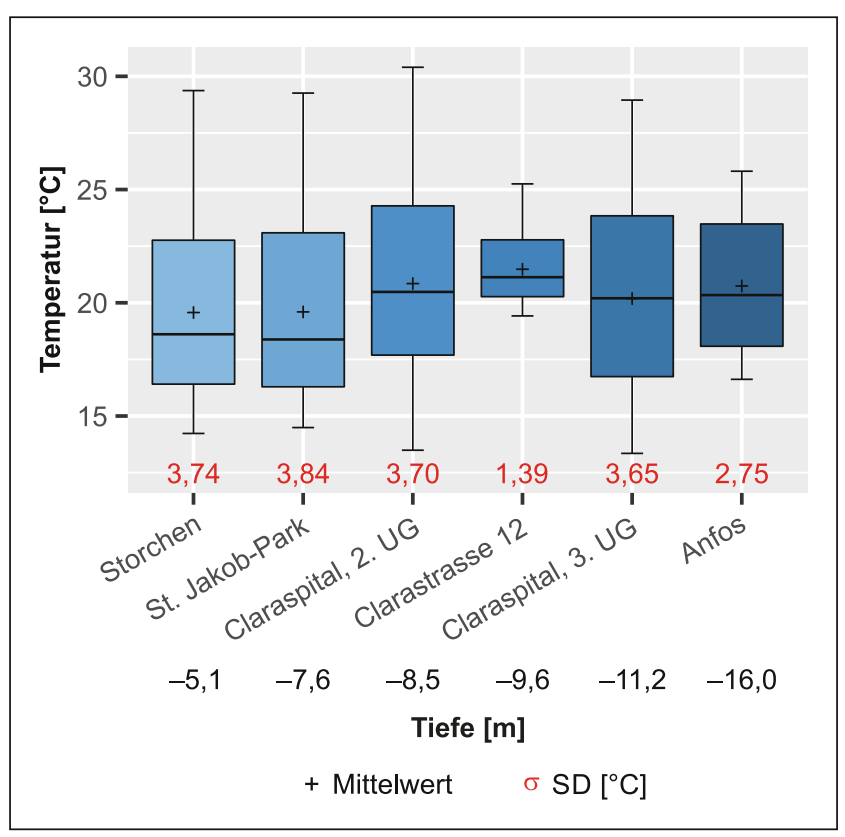

Abb. 4 Boxplot-Darstellung der $\mathrm{T}_{\mathrm{TG}}$-Verteilung nach Standorttiefe Fig. 4 Boxplot representation of the $\mathrm{T}_{\mathrm{TG}}$-distribution by site depth

zeichnenden Temperatur-Peaks ebenfalls aus, sie ersetzt ein Tagesgang mit schwacher Fluktuation.

Die Temperaturschwankungen in der Tiefgarage Clarastrasse sind kaum vergleichbar mit denjenigen der Tiefgarage Storchen (Abb. 5). Unterbrochen von einigen Ausreißern zeigt sich, dass Temperaturschwankungen in wärmeren Wochen stärker ausfallen als in kälteren. Aufgrund der mehrheitlich privaten Nutzung der Tiefgarage bleibt der verkaufsoffene Sonntag ohne Auswirkung auf $\mathrm{T}_{\mathrm{TG}}$. Weihnachten und Neujahr sind nicht von anderen Tagen zu un- terscheiden und auch die ausgewählte Woche während des „Lockdowns“ unterscheidet sich kaum von anderen Wochen.

Die beiden Messungen in der Tiefgarage des St. Claraspitals zeigen einige Unterschiede (Abb. 5). Während sich im 2. UG, welches überwiegend von Besuchenden der Patienten genutzt wird, auch an Sonn- und einigen Feiertagen, wie Christi Himmelfahrt und dem 1. August, TemperaturPeaks entwickeln, bleibt die Temperatur im Stockwerk darunter, welches v. a. dem Personal zur Verfügung steht, meist über das gesamte Wochenende, ebenso wie an Feiertagen, vergleichsweise konstant. Da das St. Claraspital als Gesundheitseinrichtung nicht dem „Lockdown“ unterlag, sind in dieser Zeit im 3. UG keine ungewöhnlichen Abweichungen der Temperatur zu beobachten, im 2. UG fallen die täglichen Schwankungen aufgrund der strengeren Regeln für Besuchende etwas tiefer aus.

Die Tiefgarage St. Jakob-Park ist wie die Tiefgarage Storchen von umliegenden Geschäften und einem angrenzenden Shoppingcenter geprägt. Ein Tagesgang, welcher von Montag bis Samstag deutlich zu erkennen ist, fällt an Sonn- und gesetzlichen Feiertagen komplett weg. Der verkaufsoffene Sonntag zeigt eine deutliche Temperaturerhöhung. In der Zeit des „Lockdowns“, in welcher bis auf einen Lebensmittelladen alle Geschäfte des Shoppingcenters schließen mussten, verschwinden die Temperaturschwankungen fast gänzlich.

Der Temperaturverlauf in der Tiefgarage Anfos lässt sich am ehesten mit jenem in der Tiefgarage Clarastrasse vergleichen, obwohl der Unterschied zwischen Wochen- und Wochenendtagen hier noch stärker ausfällt, v. a. in Wochen mit höheren Temperaturen. Die Tiefgarage ist zwar generell durch die Öffentlichkeit nutzbar, das Stockwerk, in

Tab. 4 Pearson-Korrelationskoeffizienten $\mathrm{r}$ der Stundenwerte und Tagesmittel mit zeitlicher Verzögerung der höchsten erreichten Werte; Standorttiefe; mittlere Abweichung vom gleitenden Tagesmittel

Table 4 Pearson-correlation-coefficient $r$ of hourly values and daily mean with time delay of the highest values reached; site depth; mean deviation from the moving daily mean

\begin{tabular}{|c|c|c|c|c|c|c|}
\hline Standort & $\begin{array}{l}\text { Tiefe Messystem } \\
\text { unter GOK }[\mathrm{m}]\end{array}$ & $r_{h}^{\mathrm{a}}[-]$ & $r_{\bar{d}}^{\mathrm{b}}[-]$ & $t r_{\max }^{\mathrm{c}}[\mathrm{h}]$ & $\overline{\Delta T_{\text {GWfloat }}}{ }^{d}\left[{ }^{\circ} \mathrm{C}\right]$ & $\sigma^{\mathrm{e}}\left[{ }^{\circ} \mathrm{C}\right]$ \\
\hline 1: Storchen & $-5,1$ & 0,47 & 0,52 & +24 & 2,1 & 3,74 \\
\hline 2: Clarastrasse & $-9,6$ & 0,36 & 0,45 & $+48-72$ & 0,1 & 1,39 \\
\hline $\begin{array}{l}\text { 3: St. Claraspital, } \\
\text { 2. UG }\end{array}$ & $-8,5$ & 0,57 & 0,60 & +24 & 3,7 & 3,70 \\
\hline $\begin{array}{l}\text { 3: St. Claraspital, } \\
\text { 3. UG }\end{array}$ & $-11,2$ & 0,53 & 0,57 & +48 & 3,2 & 3,65 \\
\hline 4: St. Jakob-Park & $-7,4$ & 0,32 & 0,35 & +24 & 2,6 & 3,84 \\
\hline 5: Anfos & $-16,0$ & 0,22 & 0,23 & +96 & 1,1 & 2,75 \\
\hline
\end{tabular}

GOK Geländeoberkante

aKorrelationskoeffizient der stündlichen Messungen $r_{h}$

${ }^{\mathrm{b}}$ Korrelationskoeffizient der Tagesmittel $r_{\bar{d}}$

${ }^{c}$ Verzögerung bis zum Eintreten des grössten Korrelationkoeffizienten $t r_{\text {max }}$

${ }^{\mathrm{d}}$ Durchschnittliche Abweichung von $\mathrm{T}_{\mathrm{TG}}$ zum gleitenden Mittelwert $\Delta T_{\mathrm{GWfloat}}$

${ }^{\text {e}}$ Standardabweichung $\sigma$ 


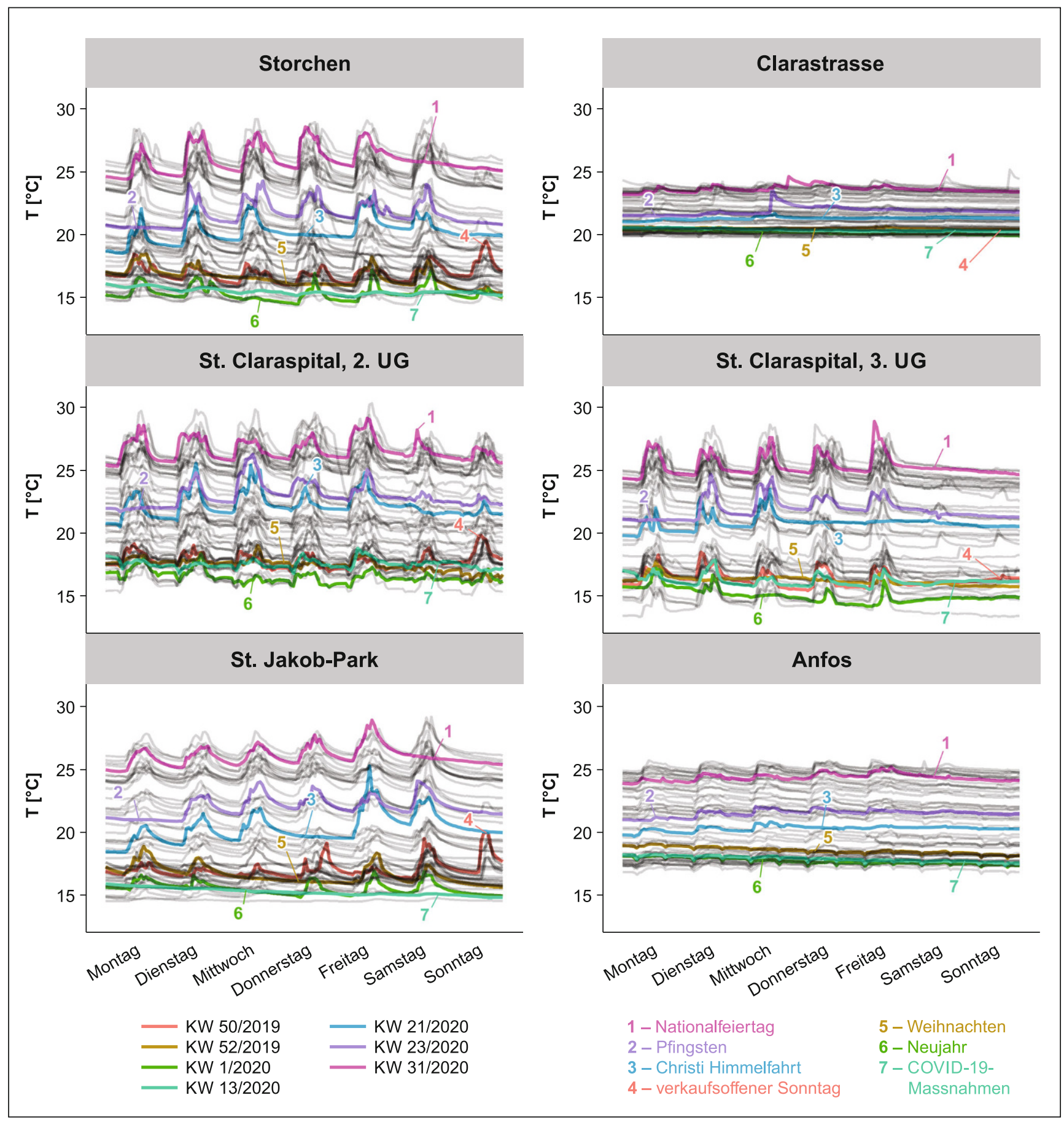

Abb. $5 \mathrm{~T}_{\mathrm{TG}}$-Tagesgänge der Tiefgarage für verschiedene ausgewählte Kalenderwochen, Wochen bzw. Tage mit Besonderheiten sind hervorgehoben und gekennzeichnet

Fig. 5 Daily progression of $\mathrm{T}_{\mathrm{TG}}$ of the underground parkings for different selected calendar weeks, weeks or days with special features are highlighted and marked

welchem die Messungen erfasst wurden, ist jedoch durch ein geringes Verkehrsaufkommen gekennzeichnet. Fehlende Temperaturmaxima an Pfingsten und Christi Himmelfahrt sind sehr leicht ausgeprägt, in Wochen, welche durch kalte Temperaturen geprägt sind, sind keine täglichen Ausschläge nach oben mehr zu erkennen. Stattdessen wird ein regelmäßiges Muster im Verlauf von $\mathrm{T}_{\mathrm{TG}}$ erkennbar, nach welchem die Temperatur zweimal pro Tag um ca. 0,1 bis $0,2^{\circ} \mathrm{C}$ absinkt. Nach Angaben des technischen Dienstes der Tiefgarage handelt es sich dabei um die Einflüsse einer Lüftungsanlage, welche in einem zwölfstündigen Rhythmus kurzzeitig eingeschaltet wird, um Frischluft in die Tiefga- 
rage zu leiten. Die kalten Außentemperaturen im Winter sorgen für eine kurzzeitige Abkühlung in der Tiefgarage.

Zusammenfassend lassen sich folgende Erkenntnisse ableiten: (1) je höher die Ausgangstemperatur desto höher ist auch die Ausprägung täglicher Temperaturschwankungen; (2) das Ausbleiben von Temperaturausschlägen an Sonnund Feiertagen in den Tiefgaragen, welche ansonsten die ausgeprägtesten Tagesgänge zeigen, deuten darauf hin, dass hier die Nutzung, also das Einstellen aufgeheizter Fahrzeuge und der Betrieb von Heiz- und Kühlanlagen, den stärksten Einfluss auf die Temperaturen hat.

Wie bereits beschrieben erklärt sich dadurch auch die Ungleichheit der Korrelationskoeffizienten von Stundenwerten und Tagesmitteln. Die nutzungsbedingte tägliche Temperaturfluktuation steht nicht in direktem Zusammenhang mit dem Tagesgang von $\mathrm{T}_{\text {meteo }}$ und resultiert somit in einer geringeren Korrelation der stündlichen Messungen. Eine erneute Bereinigung der Temperaturreihen durch das Entfernen der täglichen, ,anthropogen verursachten“ Temperatur-Peaks könnte die Korrelation für die Tagesmittelwerte weiter verbessern.

Werden die $\mathrm{T}_{\mathrm{TG}}$-Tagesmittelwerte betrachtet, gewinnt der Faktor Standorttiefe wieder an Bedeutung (Tab. 4). Mit zunehmender Tiefe der Messstation nimmt auch die Verzögerung bis zum Erreichen des größten r-Werts zu. Auch hier kann der Einflussfaktor der Tiefgaragennutzung nicht vernachlässigt werden, was sich erneut am Beispiel der Tiefgarage des St. Claraspitals zeigen lässt: $\operatorname{Im} 3$. UG $(-11,2 \mathrm{~m})$ wird der höchste r-Wert nach $48 \mathrm{~h}$ festgestellt, in der höher gelegenen Tiefgarage Clarastraße $(-9,6 \mathrm{~m})$ zwischen 48 und $72 \mathrm{~h}$, im wiederum höheren 2. UG der Tiefgarage des St. Claraspitals $(-8,5 \mathrm{~m})$ nach $24 \mathrm{~h}$. Dementsprechend ist eine Kombination der Faktoren Tiefe und Nutzungsintensität für die Reaktionsdauer des $\mathrm{T}_{\mathrm{TG}}$-Tagesmittelverlaufs verantwortlich. Die deutlich größeren Verzögerungen von bis zu $96 \mathrm{~h}$ treten in den wenig genutzten Tiefgaragen auf, was darauf hindeutet, dass auch die Reaktionszeiten hier, entsprechend ihrer Tiefe, ohne die nutzungsbedingten Temperaturschwankungen länger ausfallen würden.

Zusammenfassend zeigen die Korrelationsanalysen den Zusammenhang der meteorischen Lufttemperatur mit jener in den Tiefgaragen. Dabei zeigt der Temperaturverlauf in den Tiefgaragen eine klare Abhängigkeit von der Nutzungsart: In Tiefgaragen mit höherem Aufkommen täglicher Einund Ausfahrten konnten größere tägliche Temperaturanstiege nachgewiesen werden, durch welche Unterschiede von bis zu $2^{\circ} \mathrm{C}$ in den Tagesmittelwerten zu verzeichnen sind. Besonders deutlich konnte dies im Zeitraum des „Lockdowns" während der COVID-19-Pandemie zwischen März und Mai 2020 beobachtet werden. So blieben für Tiefgaragen, welche durch die Öffentlichkeit genutzt werden (z.B. das Parking eines Shoppingcenters), regelmäßig eintretende tägliche Temperaturschwankungen aus. Wenig Veränderun- gen konnten für Tiefgaragen, welche privat genutzt oder Teil einer essenziellen Einrichtung (z. B. angeschlossen an ein Spital) waren, verzeichnet werden.

\section{Einfluss von $\mathrm{T}_{\mathrm{TG}}$ auf $\mathrm{T}_{\mathrm{GW}}$}

In den Temperaturzeitreihen ist deutlich zu erkennen, dass $\mathrm{T}_{\mathrm{TG}}$ mehrheitlich $\mathrm{T}_{\mathrm{GW}}$ überschreiten. Zur genaueren Analyse der Temperaturunterschiede wurde $\Delta \mathrm{T}$ der Tagesmittel von $T_{T G}$ und $T_{G W}$ sowie den $T_{G W s i m}$ als Flächendiagramme dargestellt (Abb. 6). Positive Werte deuten darauf hin, dass $\mathrm{T}_{\mathrm{TG}}$ die $\mathrm{T}_{\mathrm{GW}}$ überschreitet und dementsprechend die Tiefgarage Wärme nach außen emittiert; bei negativen Werten unterschreitet $\mathrm{T}_{\mathrm{TG}}$ die $\mathrm{T}_{\mathrm{GW}}$, die Tiefgarage absorbiert Wärme. Um die Schwankungen von $\mathrm{T}_{\mathrm{GW}}$ und $\mathrm{T}_{\mathrm{GW} \text { sim }}$ in den Vergleich miteinzubeziehen werden diese Temperaturkurven ebenfalls als Referenz dargestellt.

Abb. 6 zeigt für den Standort Storchen die oft großen saisonalen Unterschiede in den Winter- und Frühlingsmonaten. $\Delta \mathrm{T}$, abgeleitet aus $\mathrm{T}_{\mathrm{TG}}$ in Relation zu $\mathrm{T}_{\mathrm{GW}}(\mathrm{GWM}$ 1052) und $T_{G W s i m}$, schwankt zwischen 3 und $-1{ }^{\circ} \mathrm{C}$. Wärmeemission und -absorption gleichen sich bis Ende April mit einer durchschnittlichen Temperatur von ca. $16,2{ }^{\circ} \mathrm{C}$ in etwa aus. In den Sommermonaten wird $\Delta \mathrm{T}$ deutlich größer, fällt nicht mehr in den negativen Bereich und erreicht ein Maximum von $8,2^{\circ} \mathrm{C}$ über $\mathrm{T}_{\mathrm{TG}}$. $\mathrm{T}_{\mathrm{GWsim}}$ bildet über die Zeit eine sinusförmige Kurve mit einer Amplitude von $0,9^{\circ} \mathrm{C}$, mit einem lokalen Maximum im Mai, einem lokalen Minimum im Februar und einem weiteren Maximum bei $19,1^{\circ} \mathrm{C}$ im September.

Im Vergleich zum Standort Storchen zeigen die Temperaturschwankungen der Tiefgarage Clarastrasse eine deutlich geringere Fluktuation. $\Delta \mathrm{T}$ variiert hier zwischen $3,6^{\circ} \mathrm{C}$ im Winter und $8,4^{\circ} \mathrm{C}$ im Sommer, bleibt also stets im positiven Bereich. Der $\mathrm{T}_{\mathrm{GWsim}}$-Mittelwert beträgt $15,8^{\circ} \mathrm{C}$. Ähnlich sieht es für die beiden GWM aus: An GWM Waldshuterstrasse (1064) wurde mit durchschnittlich $14,0^{\circ} \mathrm{C}$ eine tiefe $\mathrm{T}_{\mathrm{GW}}$ gemessen, $\Delta \mathrm{T}$ liegt zwischen 5,7 und $10,2{ }^{\circ} \mathrm{C}$; die $\mathrm{T}_{\mathrm{GW}}$ an GWM Dolderweg (1161) liegt dazwischen, mit einem Mittelwert von $14,7^{\circ} \mathrm{C}$ und einer Variation zwischen 5,0 und $9,5^{\circ} \mathrm{C}$.

Die in unterschiedlichen Tiefen gemessenen $\mathrm{T}_{\mathrm{TG}}$ in der Tiefgarage des St. Claraspitals unterscheiden sich untereinander, wie in den Temperaturzeitreihen bereits zu erkennen war (Abb. 2). Ein $\Delta \mathrm{T}$ von $1,3{ }^{\circ} \mathrm{C}$ zwischen dem 2. und 3. UG bewirkt, dass das mit $\mathrm{T}_{\mathrm{GW} \text { sim }}$ abgeleitete $\Delta \mathrm{T}$ im unteren Stockwerk im Januar kurzzeitig negative Werte annimmt, mit einem Minimum von $-1,5^{\circ} \mathrm{C}$. Eine in etwa ausgeglichene Bilanz zwischen Wärmeemission und -absorption besteht aber nur im Januar; unmittelbar vorher und direkt anschließend nehmen die Temperaturen wieder zu und $\Delta \mathrm{T}$ erreicht Maximalwerte von $11,3^{\circ} \mathrm{C}$. Gleiche Werte erreicht $\Delta \mathrm{T}$ für die GWM Magdenweglein 46 (GWM 3966), an der 


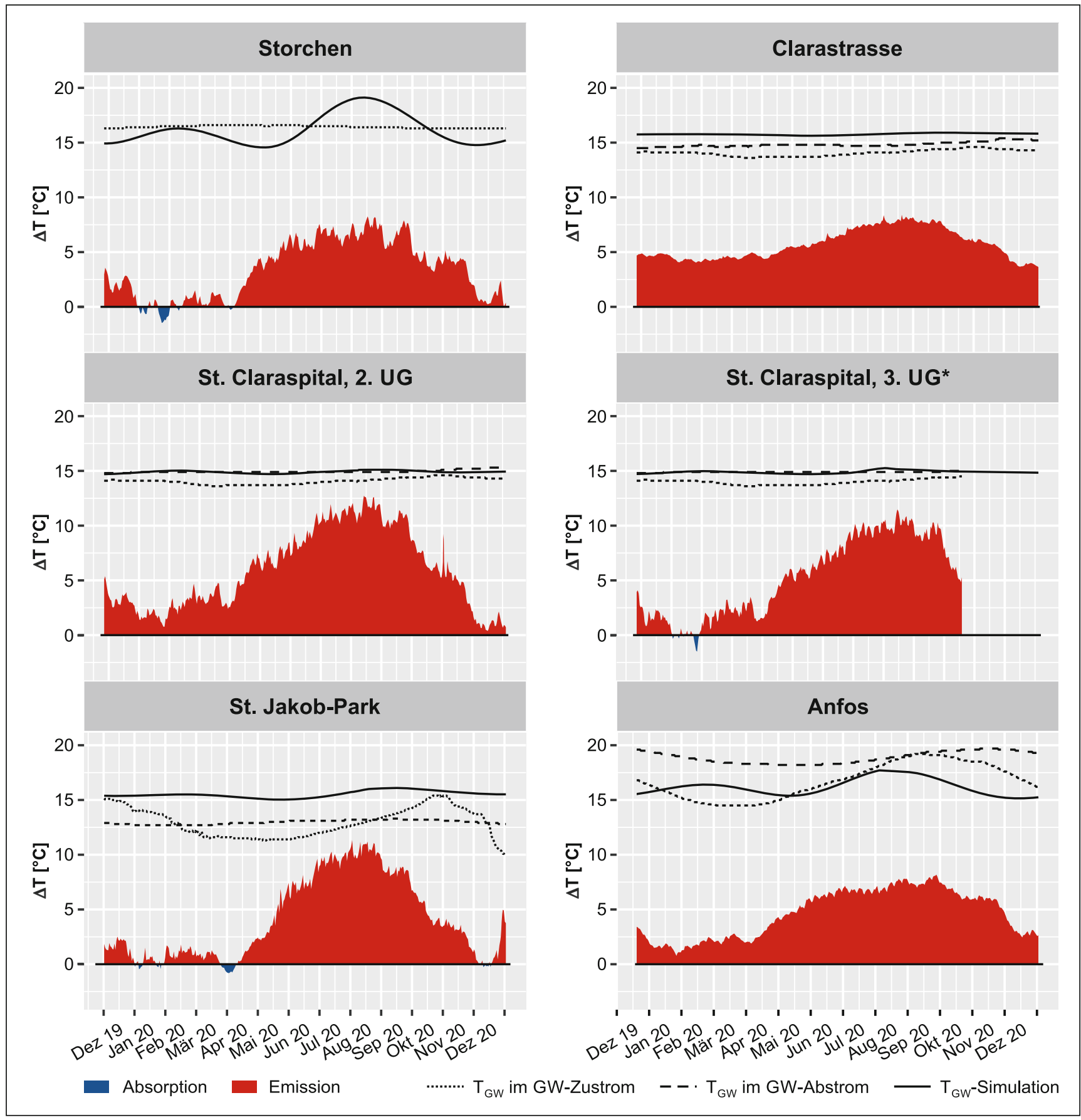

Abb. $6 \Delta \mathrm{T}$ zwischen $\mathrm{T}_{\mathrm{TG}}$ und $\mathrm{T}_{\mathrm{GW}}$ sowie Temperaturzeitreihen $\mathrm{T}_{\mathrm{GW}}$ und $\mathrm{T}_{\mathrm{GWsim}}$ (* Daten nur bis Mitte Oktober) Fig. $6 \Delta \mathrm{T}$ between $\mathrm{T}_{\mathrm{TG}}$ and $\mathrm{T}_{\mathrm{GW}}$ and temperature time series $\mathrm{T}_{\mathrm{GW}}$ and $\mathrm{T}_{\mathrm{GW}}$ sim $(*$ data only until mid-October)

GWM Waldshuterstrasse (1064) sind diese mit $12,5^{\circ} \mathrm{C}$ noch höher. Die höhere Temperatur im 2. UG führt dazu, dass $\Delta \mathrm{T}$ hier gänzlich im positiven Bereich liegt, also permanent Wärme von der Tiefgarage nach außen emittiert wird. Die größten $\Delta \mathrm{T}$-Werte liegen hier bei 12,8 (GWM 3966) bzw. $13,5^{\circ} \mathrm{C}$ (GWM 1064). Der Messpunkt im 2. UG liegt oberhalb des Grundwasserpegels, hier wird die Wärme an das umliegende Lockergestein und nicht an das Grundwas- ser abgegeben. Im Umkehrschluss bedeutet dies, dass auch kein Grundwasser auf Höhe des 2. UG fließt, welches die Wärme aufnehmen und wegtransportieren kann (advektiver Wärmetransport); ein weiterer Faktor, neben der verstärkten Nutzung, der den Temperaturunterschied zwischen den Untergeschossen erklärt.

Für die Tiefgarage St. Jakob-Park nimmt das mit $\mathrm{T}_{\mathrm{GW} \text { sim }}$ abgeleitete $\Delta \mathrm{T}$ in den Winter- und Frühlingsmonaten ver- 
Tab. 5 Verhältnis der Anzahl Tage $n$ mit positiven und negativem $\Delta \mathrm{T}$ sowie über den Zeitraum

Table 5 Ratio of the number of days $n$ with positive and negative $\Delta \mathrm{T}$ and over the period

\begin{tabular}{lllll}
\hline Standort & $n$ & $n$ & $n_{\text {total }}$ & Verhältnis \\
& $\Delta \mathrm{T}<0$ & $\begin{array}{l}\Delta \mathrm{T}>0 \\
{[\#]}\end{array}$ & $\begin{array}{l}n_{\text {total }} \\
{[\#]}\end{array}$ \\
\hline 1: Storchen & 32 & 334 & 366 & $\sim 0,09$ \\
2: Clarastrasse & 0 & 366 & 366 & - \\
$\begin{array}{l}\text { 3: St. Claraspi- } \\
\text { tal, 2. UG }\end{array}$ & 0 & 366 & 366 & - \\
3: St. Claraspi- & 13 & 311 & 324 & $\sim 0,04$ \\
tal, 3. UG & & & & \\
4: St. Jakob- & 28 & 338 & 366 & $\sim 0,07$ \\
Park & & & & \\
5: Anfos & 0 & 366 & 366 & $\sim 0,52$ \\
Nordtangente & 191 & 174 & 365 & \\
\hline
\end{tabular}

gleichsweise kleine Werte an. Anfang April wurden bei einem lokalen Temperaturminimum sogar negative Werte verzeichnet; mit dem Ansteigen der Temperaturen in den Sommermonaten nimmt auch $\Delta \mathrm{T}$ wieder zu und erreicht Werte bis $11,3^{\circ} \mathrm{C}$. Der Mittelwert beträgt $15,3^{\circ} \mathrm{C}$. Die $\mathrm{T}_{\mathrm{GW}^{-}}$ Mittelwerte betragen an der GWM G80-Karussell (20J96) 13,0, an GWM Redingstrasse (749) $12,8^{\circ} \mathrm{C}$, allerdings unterscheiden sich die $\Delta \mathrm{T}$-Werte aufgrund der verschiedenen Verlaufskurven; $\mathrm{T}_{\mathrm{GW}}$ in GWM 749 sinkt dabei über die Zeit um $3,6^{\circ} \mathrm{C}$, weshalb die $\Delta \mathrm{T}$-Werte im Winter deutlich tiefer ausfallen als im Sommer. $\mathrm{T}_{\mathrm{GW}}$ in GWM 749 übersteigt $\mathrm{T}_{\mathrm{TG}}$ in den Sommermonaten um bis zu $14,1^{\circ} \mathrm{C}$, die $\mathrm{T}_{\mathrm{GW}}$ in GWM 20J96 um maximal $13,9^{\circ} \mathrm{C}$.

Die Darstellungen in Abb. 6 zeigen, dass $\mathrm{T}_{\mathrm{TG}}$ während überwiegender Zeit höher sind als jene des umgebenden Grundwassers. Vor allem in den Sommermonaten sind sehr hohe Temperaturunterschiede von bis über $14^{\circ} \mathrm{C}$ zu beobachten. Auch im Winter und Frühling sinkt $\Delta \mathrm{T}$ selten in den negativen Bereich und dies auch nur, wenn bereits deutlich erhöhte $\mathrm{T}_{\mathrm{GW}}$ vorliegen. Die Wärmeabsorption kann also die -emissionen der Tiefgaragen nicht ausgleichen; über den Zeitraum, für den $\mathrm{T}_{\mathrm{GW}}$-Messungen vorliegen, beläuft sich die höchste Anzahl von Tagen mit negativem $\Delta \mathrm{T}$-Wert auf 32 (Tab. 5).

Zusammenfassend zeigen die Vergleiche zwischen den Temperaturunterschieden in den Tiefgaragen, den Temperaturmessungen in nahegelegenen Grundwassermessstellen und den Resultaten einer Wämetransportmodellierung den Zusammenhang von Einwirkungen der Nutzungsintensität der Tiefgaragen. In Abhängigkeit von Gebäudetiefe, Grundwasserspiegelhöhe und -fließgeschwindigkeiten konnten die Wärmelasten für die ausgewählten Tiefgaragen quantitativ abgeschätzt werden. Dabei zeigte sich, dass bereits signifikante Unterschiede von mehr als $1{ }^{\circ} \mathrm{C}$ entstehen, je nachdem, ob ein Stockwerk im grundwassergesättigten Bereich des Untergrunds liegt oder in der grundwasserungesättigten Zone. V.a. auch die Flächengröße, welche in Kontakt mit dem Grundwasser steht, spielt eine große
Rolle, wie viel Wärme in das Grundwasser abgegeben wird.

\section{Vergleich Tiefgaragen - Autobahntunnel Nordtangente}

Im Gegensatz zu den Tiefgaragen liegen die Temperaturen in den Tunneln der Nordtangente an durchschnittlich 191 Tagen der einjährigen Messperiode unter der mittleren $\mathrm{T}_{\mathrm{GW}}$, also während ca. 52\% der Zeit. In den Winter- und teilweise auch in den Herbst- und Frühlingsmonaten wurden Temperaturen gemessen, die die mittlere $\mathrm{T}_{\mathrm{GW}}$ deutlich um bis $\mathrm{zu} 15^{\circ} \mathrm{C}$ unterschreiten. In diesen Monaten wird demnach Wärme aus dem Grundwasser durch die Untergrundstruktur absorbiert. In den Sommermonaten kehrt sich die Situation, die Temperaturen steigen um bis zu $17,8^{\circ} \mathrm{C}$ über die mittleren $\mathrm{T}_{\mathrm{GW}}$, während eines Zeitraums von durchschnittlich 174 Tagen wird Wärme von den Tunnelbauwerken an den Grundwasserkörper abgegeben (Tab. 5).

Es entsteht also über das gesamte Jahr hinweg ein Gleichgewicht zwischen Wärmeemission und -absorption, welches in den Tiefgaragen nicht zu erkennen ist. Die Gebäudeform der Untergrundstruktur spielt also eine wesentliche Rolle im Wärmehaushalt: Die offene Struktur des Tunnelbauwerkes lässt vor allem über die Tunnelportale einen deutlich größeren Austausch mit der Außenlufttemperatur und demnach ausgeprägtere Temperaturschwankungen über die Jahreszeiten zu als die eher geschlossene, kompaktere Form der Tiefgarage. Im Tunnel besteht zudem eine ständige Bewegung der Fahrzeuge sowie eine zusätzliche künstliche Ventilation, welche den Luftaustausch fördert. Dagegen werden die Fahrzeuge in den Tiefgaragen meist abgestellt, wo sie abkühlen und Wärme an die Umgebung abgeben. 


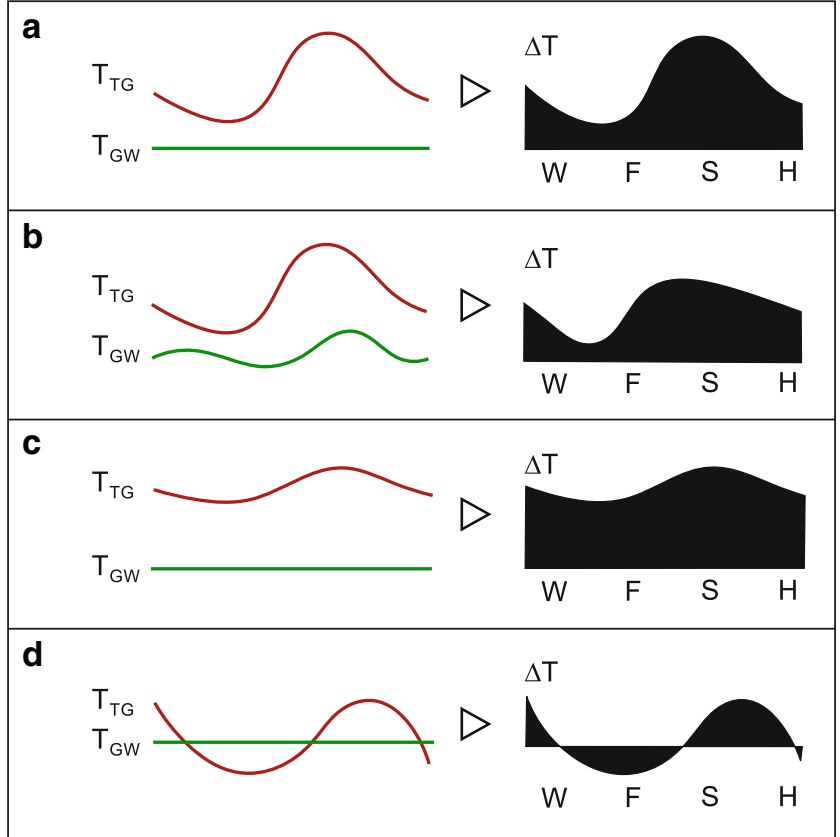

Abb. 7 Das Verhältnis von $\mathrm{T}_{\mathrm{TG}} \mathrm{zu} \mathrm{T}_{\mathrm{GW}}$ resultiert in 4 verschiedenen $\Delta \mathrm{T}$-Profilen, abhängig von den jeweiligen Temperaturverläufen über die Zeit; Tiefgaragen a St. Claraspital und St. Jakob-Park, b Storchen und Anfos, $\mathbf{c}$ Clarastrasse und Tunnelbauwerk d Nordtangente

Fig. 7 The ratio of $\mathrm{T}_{\mathrm{TG}}$ to $\mathrm{T}_{\mathrm{GW}}$ results in 4 different $\Delta \mathrm{T}$ profiles, depending on the respective temperature curves over time; underground car parks a St. Claraspital and St. Jakob-Park, b Storchen and Anfos, c Clarastrasse, and tunnel structure d Nordtangente

\section{Konzept $\Delta T$-Profile für den thermischen Einfluss von Untergrundstrukturen}

Für die untersuchten Untergrundstrukturen, einschließlich der Tiefgaragen und des Autobahntunnels Nordtangente, können vier verschiedene thermische Einwirkungsmuster auf das Grundwasser abgeleitet werden (Abb. 7):

1. Tiefgaragen St. Claraspital und St. Jakob-Park: $\mathrm{T}_{\mathrm{TG}}$ mit hoher Fluktuation, tief im Winter und hoch im Sommer; $\mathrm{T}_{\mathrm{GW}}$ verläuft tief und flach. $\Delta \mathrm{T}$ im Winter mit kleinen/ negativen Werten, die im Verlauf des Jahres steil zu- und wieder abnehmen.

2. Tiefgaragen Storchen und Anfos: $\mathrm{T}_{\mathrm{TG}}$ mit hoher Fluktuation, tief im Winter und hoch im Sommer; $T_{\mathrm{GW}}$ verläuft hoch, mit versetzter Temperaturkurve $\mathrm{zu}_{\mathrm{TG}} . \Delta \mathrm{T}$ zu Beginn im Winter mit kleinen/negativen Werten, die im Jahresverlauf steil zu- und stetig wieder abnehmen.

3. Tiefgarage Clarastrasse: $T_{\mathrm{TG}}$ mit geringer Fluktuation, hoch im Winter und noch höher im Sommer; $\mathrm{T}_{\mathrm{GW}}$ verläuft hoch und flach. $\Delta \mathrm{T}$ durchgehend mit mittleren, im Verlauf leicht fluktuierenden Werten.

4. Tunnelbauwerk Nordtangente: Temperatur der Tunnelinnenluft mit sehr hoher, saisonaler Fluktuation; $\mathrm{T}_{\mathrm{GW}}$ verläuft hoch und flach (Abb. 3). $\Delta \mathrm{T}$ nimmt im Gegensatz zu den Tiefgaragen über größere Zeiträume negative Werte an.

\section{Wärmelast der Untergrundstrukturen}

Die Größenordnung der Wärmelast, die von den unterirdischen Gebäudestrukturen sowohl in die ungesättigte als auch die gesättigte Zone entweicht, kann mit einer einfachen Berechnung quantitativ abgeschätzt werden (Epting et al. 2013). Unter Berücksichtigung der Gebäudefläche $A_{G W}$, welche mit dem Grundwasser der umliegenden gesättigten bzw. dem Gestein/Boden der ungesättigten Zone in Kontakt steht, des Wärmedurchgangskoeffizienten $k$ der Gebäudeaußenwände und des Temperaturunterschieds $\Delta \mathrm{T}$ zwischen der Temperatur in der Untergrundstruktur und den Grundwassertemperaturen im Zustrom (in K) kann der Wärmeaustausch $E$ berechnet werden:

$E=k \cdot A_{G W} \cdot \Delta T$.

Das älteste der untersuchten Gebäude ist die Tiefgarage Storchen (Baujahr 1959), weshalb basierend auf dem Normenwerk des Schweizerischen Ingenieur- und Architektenvereins (SIA 380/1) für alle Tiefgaragen eine gute Gebäudeisolation und demnach ein Wärmedurchgangskoeffizient $\mathrm{k}$ von rund $0,3 \mathrm{~W} \mathrm{~m} \mathrm{~m}^{-2} \mathrm{~K}^{-1}$ angenommen werden kann. Ausgehend von den Daten zu Gebäudegrundflächen und den gemessenen Stockwerkshöhen lassen sich diese Berechnungen auf die Standorte dieser Arbeit übertragen. Dazu wurden die jeweiligen Flächen der Seitenwände der Tiefgaragen, die unterhalb des Grundwasserspiegels liegen, berechnet. In den Fällen, in welchen die Gebäudeunterkante ebenfalls im Grundwasser liegt und nicht in den anstehenden Fels gebaut wurde, werden die Grundfläche addiert und die Gesamtflächen mit den Wärmelasten pro $\mathrm{m}^{2}$ verrechnet. Für jede Tiefgarage konnten somit die Wärmelasten des $\Delta \mathrm{T}$-Gesamtmittelwerts sowie vergleichsweise kleinere $\Delta \mathrm{T}$ Werte der Winter- und größere $\Delta \mathrm{T}$-Werte der Sommermonate berechnet werden (Tab. 6), um saisonale Unterschiede aufzeigen zu können.

Tab. 6 und Abb. 8 zeigen, dass das Ausmaß des Wärmeaustausches nicht zwangsläufig mit der Größe des Gebäudes zusammenhängt, sondern mit der Kontaktfläche zur grundwassergesättigten Zone. Die Resultate der potenziellen Wärmeflüsse der Tiefgaragen sind modellhaft zu betrachten, da zusätzliche beeinflussende Faktoren auf die Temperaturen wie die Auswirkung des Grundwasserregimes nicht berücksichtigt wurden.

Es ist schwierig, die Wärmelasten verschiedener Städte mit unterschiedlichen städtischen Strukturen miteinander zu vergleichen. Die in Epting et al. (2017b) abgeleiteten Wärmelasten von Untergrundstrukturen in Basel liegen 
Tab. 6 Wärmeaustausch der Tiefgaragen und des Autobahntunnels Nordtangente mit dem Grundwasserkörper, normiert auf $\mathrm{m}^{2}\left(\mathrm{E}_{\text {norm }}\right)$ und für die gesamte Gebäudekontaktfläche zum Grundwasser (Eges)

Table 6 Heat exchange of the underground parkings and the Nordtangente freeway tunnel with the groundwater body, standardized to $\mathrm{m}^{2}$ ( $\left.\mathrm{E}_{\text {norm }}\right)$ and for the entire building contact surface with the groundwater (Eges)

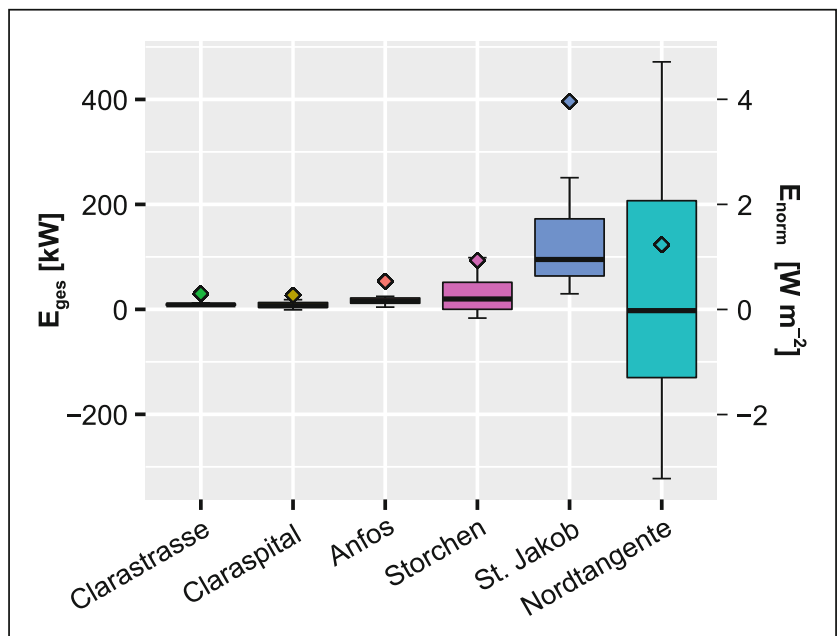

Abb. 8 Boxplot-Darstellung der gesamten $\mathrm{E}_{\mathrm{ges}}(\mathrm{kW})$ und normierten $\mathrm{E}_{\text {norm }}\left(\mathrm{W} \mathrm{m}^{-2}\right)$ Wärmelast der Untergrundstrukturen sortiert nach ansteigender Kontaktfläche zum Grundwasserkörper: Clarastrasse $\left(4041 \mathrm{~m}^{2}\right)$, Claraspital $\left(4905 \mathrm{~m}^{2}\right)$, Anfos $\left(12.155 \mathrm{~m}^{2}\right)$, Storchen $\left(29.995 \mathrm{~m}^{2}\right)$, St. Jakob $\left(59.802 \mathrm{~m}^{2}\right)$ und Nordtangente $\left(111.000 \mathrm{~m}^{2}\right)$

Fig. 8 Boxplot representation of the total $\mathrm{E}_{\mathrm{ges}}(\mathrm{kW})$ and standardized $E_{\text {norm }}\left(\mathrm{W} \mathrm{m}^{-2}\right)$ heat load of the subsurface structures, sorted by increasing contact area to the groundwater body: Clarastrasse $\left(4041 \mathrm{~m}^{2}\right)$, Claraspital $\left(4905 \mathrm{~m}^{2}\right)$, Anfos $\left(12,155 \mathrm{~m}^{2}\right)$, Storchen $\left(29,995 \mathrm{~m}^{2}\right)$, St. Jakob $\left(59,802 \mathrm{~m}^{2}\right)$ and Nordtangente $\left(111,000 \mathrm{~m}^{2}\right)$

zwischen 0,2 und $0,9 \mathrm{~W} \mathrm{~m}^{-2}$, abhängig von der Lage der Strukturen innerhalb des Aquifers sowie von den hydraulischen und baulichen Randbedinungen. In Menberg et al. (2013a) resultierten aus einer räumlichen Analyse der Wärmelasten von Untergrundstrukturen Werte im Bereich von $-0,1$ und $>10 \mathrm{~W} \mathrm{~m}^{-2}$. In Benz et al. (2015) werden anthropogene Wärmelasten von Untergrundstrukturen in der GröBenordnung von $3,61 \pm 3,37 \mathrm{~W} \mathrm{~m}^{-2}$ für Karlsruhe und von $0,57 \pm 0,47 \mathrm{~W} \mathrm{~m}^{-2}$ für Köln vorgestellt. Rees et al. (2000) und Thomas und Rees (1999) dokumentieren Wärmelasten durch Erdgeschossplatten von Gebäuden zwischen 0 und $20 \mathrm{Wm}^{-2}$ und Ferguson und Woodbury (2004) schätzten den Wärmeverlust unter einem Gebäude auf $\sim 2 \mathrm{~W} \mathrm{~m}^{-2}$. Die im Rahmen dieser Untersuchungen abgeleiteten Wärmeeinträ- ge liegen also durchaus im Erfahrungsbereich vorangegangener Untersuchungen.

An Orten mit großer Grundwassermächtigkeit und hohen Grundwasserfließgeschwindigkeiten ist zu erwarten, dass mehr Wärme von den Tiefgaragen abgeführt wird (advektiver Wärmetransport). Am Beispiel der Tiefgaragen Storchen und Anfos in der Großbasler Innenstadt sowie St. Claraspital am Kleinbasler Stadtrand wird dies deutlich. Die Grundwasserfließgeschwindigkeiten werden hier durch Spundwände am Rheinufer, welche die Fluss-Grundwasser-Interaktion verhindern sollen, stark abgemindert (Epting et al. 2017b). Durch die verringerte Strömung im Grundwasserkörper werden die Temperatureinträge nur langsam abgeführt, $\mathrm{T}_{\mathrm{GWsim}}$ unterscheidet sich an der Tiefgarage Storchen mit $16,2^{\circ} \mathrm{C}$, bzw. mit $16,1^{\circ} \mathrm{C}$ an der Tiefgarage Anfos nur geringfügig von $\mathrm{T}_{\mathrm{GW}}$ der GWM $1052 \mathrm{mit} 16,5^{\circ} \mathrm{C}$ im Grundwasserzustrom der Tiefgarage Storchen bzw. ist sogar tiefer als $\mathrm{T}_{\mathrm{GW}}$ mit $18,6^{\circ} \mathrm{C}$ an der GWM 3755, die im Grundwasserabstrom der Tiefgarage Anfos liegt; vor dem Rheinufer entsteht somit ein Wärmestau (Abb. 1). Im Gegensatz dazu folgt der Grundwasserfluss um die Tiefgarage St. Claraspital einem hydraulischen Gefälle von ca. $0,5 \%$. $\mathrm{T}_{\mathrm{GWsim}}$ liegt hier bei $14,9^{\circ} \mathrm{C}$ und um knapp ein Grad höher als $\mathrm{T}_{\mathrm{GW}}$ im Grundwasserzustrom; im Grundwasserabstrom bei GWM 1075, die in ihrer Position leicht lateral verschoben zum Fließrichtungsvektor des Grundwassers liegt, erreicht die Temperatur nur $14,8^{\circ} \mathrm{C}$, ist also wieder geringfügig gesunken.

\section{Schlussfolgerungen}

Eine nachhaltige thermische Bewirtschaftung urbaner Untergrundressourcen sollte eine angemessene Bewertung der thermischen Auswirkungen, einschließlich der Quantifizierung der Wärmelasten von Gebäudestrukturen, umfassen. Im Rahmen unserer Untersuchungen können wir die folgenden Schlussfolgerungen für die evaluierten Untergrundstrukturen in Basel ziehen: 
- Zu fast jedem Zeitpunkt liegt $\mathrm{T}_{\mathrm{TG}}$ über $\mathrm{T}_{\text {meteo, }}$, erst in den Frühlings- und Sommermonaten liegt $\mathrm{T}_{\text {meteo }}$ aufgrund der hohen Außenlufttemperaturen kurzzeitig über $\mathrm{T}_{\mathrm{TG}}$. In allen Temperaturzeitreihen, welche in den Tiefgaragen aufgezeichnet wurden, ist mit dem Zeitverlauf von Winter zu Sommer ein Anstieg der Temperaturen zu beobachten, je nach Standort mit unterschiedlicher Ausprägung.

- Die abgeschlossene Gebäudeform von Tiefgaragen begünstigt das Entstehen erhöhter $\mathrm{T}_{\mathrm{TG}}$, welche in einer mehr oder weniger konstanten Wärmelast in das Grundwasser resultieren. Darin unterscheiden sich Tiefgaragen von Tunnelbauwerken, die deutlich größere jahreszeitliche Temperaturunterschiede und einen vergleichsweise ausgeglicheneren Wärmeaustausch aufweisen.

- Die Resultate illustrieren den thermischen Einfluss der Untergrundstrukturen auf das Grundwasser, wobei die Temperaturen im Grundwasserabstrom der Tiefgaragen teilweise um bis zu $2,7^{\circ} \mathrm{C}$ höher sind verglichen mit jenen in den GWM gemessenen $\mathrm{T}_{\mathrm{GW}}$ im Grundwasserzustrom.

- Ein wesentlicher Einflussfaktor auf $\mathrm{T}_{\mathrm{TG}}$ ist der Wärmeeintrag von Kraftfahrzeugen, der Einflussfaktor Nutzung ist größer als jener der Standorttiefe. Dieser sorgt für eine zusätzliche Erhöhung der $\mathrm{T}_{\mathrm{TG}}$-Tagesmittel um 1 bis $2^{\circ} \mathrm{C}$.

Danksagung Hiermit möchten wir uns bei Dominik Amrein (Amt für Umwelt und Energie, Kanton Basel-Stadt), Julia Fritz (PNP Geologie \& Geotechnik AG) und Michael Freivogel (Amt für Umweltschutz und Energie, Kanton Basel-Landschaft) für die Aufbereitung und Bereitstellung von Grundwassertemperaturdaten bedanken. Weiterhin bedanken wir uns bei Dr. Roland Vogt, Dr. Robert Spirig und Dr. Christian Feigenwinter (Forschungsgruppe MCR, Universität Basel) für die Zurverfügungstellung von Meteodaten. Die Arbeit wäre nicht möglich gewesen ohne die Unterstützung der Tiefgaragenbetreibenden, einschließlich Dominik Naeff (Leiter Parkhäuser Basel-Stadt), Massimo Lizzio (Leiter Gebäudemanagement St. Claraspital), Luc Weiss (Bewirtschafter Livit AG), Daniel Zimmermann (Centerleiter Shoppingcenter St. Jakob-Park) und August Hager sowie Manuela Tschamber (Bewirtschaftende Apleona AG) und Damien Dominger (Leiter des technischen Dienstes des Anfos-Hauses). Es sei auch Thomas Schaub und NSHW gedankt für die Möglichkeit, im Autobahntunnelbauwerk Nordtangente die Temperaturmesssysteme zu installieren. Schließlich bedanken wir uns bei Dorothee Becker für das Lektorat des Textes und bei Jörg Böger für die Gestaltung des finalen Layouts der Abbildungen.

\section{Funding Open access funding provided by University of Basel}

Open Access Dieser Artikel wird unter der Creative Commons Namensnennung 4.0 International Lizenz veröffentlicht, welche die Nutzung, Vervielfältigung, Bearbeitung, Verbreitung und Wiedergabe in jeglichem Medium und Format erlaubt, sofern Sie den/die ursprünglichen Autor(en) und die Quelle ordnungsgemäß nennen, einen Link zur Creative Commons Lizenz beifügen und angeben, ob Änderungen vorgenommen wurden.

Die in diesem Artikel enthaltenen Bilder und sonstiges Drittmaterial unterliegen ebenfalls der genannten Creative Commons Lizenz, sofern sich aus der Abbildungslegende nichts anderes ergibt. Sofern das betreffende Material nicht unter der genannten Creative Commons Lizenz steht und die betreffende Handlung nicht nach gesetzlichen Vorschrif- ten erlaubt ist, ist für die oben aufgeführten Weiterverwendungen des Materials die Einwilligung des jeweiligen Rechteinhabers einzuholen.

Weitere Details zur Lizenz entnehmen Sie bitte der Lizenzinformation auf http://creativecommons.org/licenses/by/4.0/deed.de.

\section{Literatur}

Ampofo, F., Maidment, G.G., Missenden, J.F.: Review of groundwater cooling systems in London. Appl Therm Eng 26, 2055-2062 (2006)

AUE: Hydrologisches Jahrbuch 2018 Grundwassertemperatur (2019)

BAG: Medienmitteilung: Verordnung 2 über Massnahmen zur Bekämpfung des Coronavirus (COVID-19), März 2020a (2020a)

BAG: Medienmitteilung: Verordnung über Massnahmen in der besonderen Lage zur Bekämpfung der Covid-19-Epidemie (Covid-19Verordnung besondere Lage) (Massnahmenverschärfung Dezember), Dezember 2020b (2020b)

Basel-Stadt: Basel-Stadt, Statistisches A.: Statistischen Jahrbuch des Kantons Basel-Stadt (2020). Version: April 2020

Bates, B.C., Kundzewicz, Z.W., Wu, S., Palutikof, J.P.: Climate Change and Water (2008)

Benz, S.A., Bayer, P., Menberg, K., Jung, S., Blum, P.: Spatial resolution of anthropogenic heat fluxes into urban aquifers. Sci. Total. Environ. 524, 427-439 (2015)

Bitterli-Brunner, P., Fischer, H.R.: Erläuterungen zum Geologischen Atlas der Schweiz, Bl. 1067. Landeshydrologie und -geologie, Arlesheim (1988)

Bobylev, N.: Mainstreaming sustainable development into a city's master plan: a case of urban underground space use. Land Use Policy 26, 1128-1137 (2009)

Brielmann, H., Griebler, C., Schmidt, S.I., Michel, R., Lueders, T.: Effects of thermal energy discharge on shallow groundwater ecosystems. FEMS Microbiol Ecol 68, 273-286 (2009)

CEC, C.o.t.E.C.: Directive of the European Parliament and of the Council Establishing a Framework for Community Action in the Field of Water Policy: Joint Text Approved by the Conciliation Committee (2000). 1997/0067(COD). C5-0347/00

Dahlem, K.-H.: The effect of groundwater on the heat loss of building parts in contact with ground, Bauphysik / Technische Gebäudeausrüstung / Baulicher Brandschutz (2000)

Deru, M.: A Model for Ground-coupled Heat and Moisture Transfer from Buildings (2003). National Renewable Energy Laboratory

Diersch, H.J.: FEFLOW-Finite Element Modeling of Flow, Mass and Heat Transport in Porous and Fractured Media. Springer, Berlin, Heidelberg, New York (2014)

Dědeček, P., Šafanda, J., Rajver, D.: Detection and quantification of local anthropogenic and regional climatic transient signals in temperature logs from Czechia and Slovenia. Clim Change 113, 787-801 (2012)

Epting, J.: Thermal management of urban subsurface resourcesDelineation of boundary conditions. Procedia Eng (2017). https:// doi.org/10.1016/j.proeng.2017.11.133

Epting, J., Huggenberger, P.: Unraveling the heat island effect observed in urban groundwater bodies-Definition of a potential natural state. J. Hydrol. Reg. Stud. 501, 193-204 (2013)

Epting, J., Haendel, F., Huggenberger, P.: Thermal management of an unconsolidated shallow urban groundwater body. Hydrol. Earth Syst. Sci. 17, 1851-1869 (2013)

Epting, J., Scheidler, S., Affolter, A., Borer, P., Mueller, M.H., Egli, L., García-Gil, A., Huggenberger, P.: The thermal impact of subsurface building structures on urban groundwater resources-A paradigmatic example. Sci. Total Environ. 596-597, 87-96 (2017a)

Epting, J., García-Gil, A., Huggenberger, P., Vázquez-Suñe, E., Mueller, MH.: Development of concepts for the management of thermal resources in urban areas - Assessment of transferability from the Basel (Switzerland) and Zaragoza (Spain) case studies. Jour- 
nal of Hydrology 548, 697-715 (2017b). https://doi.org/10.1016/ j.jhydrol.2017.03.057

Feigenwinter, C., Schmutz, M., Vogt, R., Parlow, E.: Insights from more than ten years of $\mathrm{CO}_{2}$ flux measurements in the city of Basel, Switzerland. Int. Assoc. Urban Clim. 65, 24-32 (2017)

Ferguson, G., Woodbury, A.D.: Subsurface heat flow in an urban environment. J. Geophys. Res. Earth 109, B02402 (2004). https://doi. org/10.1029/2003JB002715

Ferguson, G., Woodbury, A.D.: Urban heat island in the subsurface. Geophys. Res. Lett. 34, L23713 (2007). https://doi.org/10.1029/ 2007GL032324

Iskander, M., Aboumoussa, W., Gouvin, P.: Instrumentation and monitoring of a distressed multistory underground parking garage. J. Perform. Constr. Facil. 15, 115-123 (2001)

Jesußek, A., Grandel, S., Dahmke, A.: Impacts of subsurface heat storage on aquifer hydrogeochemistry. Environ Earth Sci 69, 1999-2012 (2013)

Kipfer, R., Livingstone, D.M.: Water Resources and Climate Change (2008). In: News, E. (Ed.).

Menberg, K., Bayer, P., Zosseder, K., Rumohr, S., Blum, P.: Subsurface urban heat islands in German cities. Sci. Total. Environ. 442, 123-133 (2013a)

Menberg, K., Blum, P., Schaffitel, A., Bayer, P.: Long-term evolution of anthropogenic heat fluxes into a subsurface urban heat island. Environ. Sci. Technol. 47, 9747-9755 (2013b)

MeteoSchweiz, Klimanormwerte 1981-2010: Lufttemperatur 2m.

MeteoSchweiz, Klimanormwerte 1981-2010: Niederschlagssumme.
Mueller, M.H., Huggenberger, P., Epting, J.: Combining monitoring and modelling tools as a basis for city-scale concepts for a sustainable thermal management of urban groundwater resources. Sci. Total Environ. 627, 1121-1136 (2018)

Possemiers, M., Huysmans, M., Batelaan, O.: Influence of aquifer thermal energy storage on groundwater quality: a review illustrated by seven case studies from Belgium. J. Hydrol. Reg. Stud. 2, 20-34 (2014)

Rees, S.W., Adjali, M.H., Zhou, Z., Davies, M., Thomas, H.R.: Ground heat transfer effects on the thermal performance of earth-contact structures. Renew Sustain Energ Rev 4, 213-265 (2000)

Thomas, H.R., Rees, S.W.: The thermal performance of ground floor slabs-A full scale in-situ experiment. Build Environ 34, 139-164 (1999)

Tissen, C., Benz, S.A., Menberg, K., Bayer, P., Blum, P.: Groundwater temperature anomalies in central Europe. Environ. Res. Lett. 14, 104012 (2019)

Zhu, K., Blum, P., Ferguson, G., Balke, K.D., Bayer, P.: The geothermal potential of urban heat islands. Environ. Res. Lett. 5, 44002 (2010). https://doi.org/10.1088/1748-9326/5/4/044002.

Hinweis des Verlags Der Verlag bleibt in Hinblick auf geografische Zuordnungen und Gebietsbezeichnungen in veröffentlichten Karten und Institutsadressen neutral. 